\title{
Financing Public Goods and Attitudes Toward Immigration*
}

Iñigo Iturbe-Ormaetxe

Universidad de Alicante
J. Gabriel Romero

Universidad de Santiago de Chile

October 2015

\begin{abstract}
We study a model where individuals choose both the level of provision of a public good and the quota of low-skilled immigrants that are allowed into the country. Individuals can supplement the public good in the private market. Immigrants affect natives through three channels: (i) the labor market; (ii) tax collection; (iii) the quality of the public good. We find that the higher the political weight of the rich (highly skilled) is, the less tolerant the poor and the middle-class are toward immigration and the more demanding they are toward increasing public spending. The rich are the most favorable to immigration. As they have more weight, the political outcome is closer to their preferences and further from the preferences of the other groups. We use data from the European Social Survey to test the implications of our model.
\end{abstract}

JEL Classification: H41, J61, D72

Keywords: Probabilistic voting model, public goods, immigration

*We wish to thank Pedro Albarrán, Rocío Alvarez, M. Dolores Collado, Juan José Dolado, and Francisco Martínez-Mora for helpful comments. Gabriel Romero thanks Fondecyt Chile Grant 11121155 for financial support. Iñigo Iturbe-Ormaetxe thanks financial support from Ministerio de Economía y Competitividad (ECO2012-34928), Generalitat Valenciana (Prometeo/2013/037) and from Instituto Valenciano de Investigaciones Económicas (IVIE). 


\section{Introduction}

Several socioeconomic factors determine native attitudes toward immigrants and hence toward immigration policies. Native workers can be reluctant to receive more immigrants due to concerns about labor market competition from foreigners. ${ }^{1}$ Another economic issue is whether immigration causes an increased tax burden on natives. That is, whether immigrants are net beneficiaries of the welfare system (Kerr and Kerr, 2011). It is this impact that has raised recently a great deal of concern regarding immigration in several European countries, particularly due to the economic crisis. ${ }^{2}$ On top of these traditional factors that work through the labor market and the welfare state, it is also important to consider how the political process affects public opinion about immigrants. Political competition leads politicians to implement immigration policies that satisfy the interest of a majority, which in turn may foster hostile attitudes toward foreigners among those in a minority. That is, we claim that a great deal of variation in attitudes toward immigration is left unexplained by the aforementioned traditional factors. Two identical individuals (in observable characteristics) may have very different attitudes toward immigration simply because they live in regions with different types of political majorities.

The aim of this paper is to assess, theoretically and empirically, to what extent labormarket concerns, welfare state considerations, and political competition drive native attitudes toward immigration. We develop and test a model in which the interplay between immigration process, labor-market concerns, and welfare-state considerations determine the shape of native preferences regarding immigration and social (tax-expenditure) policies. Our model embeds the political economy model of public provision of private goods developed by Epple and Romano (1996) into a setting where agents also decide on immigration quotas. We consider three groups of natives who differ in their skill levels (low-, medium-, and highly skilled) and their political preferences. Natives first decide how much to supplement a public good by private purchases and, second, they choose by majority vote an immigration policy

\footnotetext{
${ }^{1}$ See Blau and Kahn (2012) for a recent survey on the impact of immigration on income distribution.

${ }^{2}$ In fact, it is the perception by natives, not the true effect, what matters. For example, Cunningham (2006) demonstrates that, contrary to popular perceptions, communities in the US with high use of emergency departments have fewer immigrants.
} 
and a tax-expenditure policy. To obtain a voting equilibrium we use a version of the probabilistic voting model by Lindbeck and Weibull (1987). ${ }^{3}$ We use this model as it guarantees the existence of a political equilibrium in multi-dimensional models. It is particularly useful when citizens can be partitioned into different groups, as is our case.

An interesting implication of our model is as follows. We find that low- and mediumskilled natives living in countries where the highly skilled group is larger than the other two groups are: (i) more willing to restrict immigration and (ii) more willing to raise taxes and spending, compared to low- and medium-skilled natives living in countries where the highly skilled is not larger than the other groups. In other words, we find greater polarization of opinions on immigration in those countries in which the highly skilled group dominates. The intuition is simple. Where the rich are decisive, the political outcome is close to their bliss point. That is, a high immigration quota and a low level of public good provision. Yet this political outcome is far away from the bliss point of the other two groups, compared to countries in which the highly skilled are not decisive.

To test these implications we use data from the 2008 wave of the European Social Survey (ESS). ${ }^{4}$ The ESS contains several questions in which individuals express their attitudes toward immigrants, together with many other individual and socioeconomic data. There is also information on attitudes toward public expenditure. Individuals answer whether or not they are willing to raise spending and taxes. ${ }^{5}$ We use the education levels of individuals as a proxy for skill. Individuals with higher education will be the highly skilled (the rich), individuals with at most secondary education will be the middle-skilled, and individuals with at most primary education will be the low-skilled. We compute the fractions that the three groups represent in each country, identifying the countries in which the highly skilled have a higher weight than the other two groups.

We want to explain individual preferences regarding both immigration and public spending. In fact, we claim that these preferences are jointly determined. The natural alternative

\footnotetext{
${ }^{3}$ See also Persson and Tabellini (2000).

${ }^{4}$ ESS Round 4: European Social Survey Round 4 Data (2008). Data file edition 4.1. Norwegian Social Science Data Services, Norway - Data Archive and distributor of ESS data.

${ }^{5}$ The reason for using only the 2008 wave is that in the other waves individuals are not asked about their attitudes regarding public expenditure.
} 
is, therefore, to use a model in which this is explicitly taken into account. Since both variables of interest are dummy variables, we estimate a bivariate Probit model. In line with the previous literature we find evidence in favor of the labor market and the welfare state hypothesis. But we also find some evidence in favor of the "political process" channel. In particular, after fitting the model we calculate for each educational group average attitudes toward immigration and spending, but differentiating between whether those individuals live in a country where the highly skilled group is larger than the others groups or not. At this stage we have controlled for a number of observable characteristics, as well as for regional dummy variables. ${ }^{6}$

With respect to the attitude toward immigration our empirical evidence is in line with our theoretical prediction for both middle-skilled and highly skilled individuals, although not for the low-skilled. Consider first middle-skilled individuals. Among those who live in countries where the highly skilled are a majority, a $41 \%$ of them are favorable to immigration, compared to a $47 \%$ among those who live where the highly skilled are not a majority. For the highly skilled, differences are much bigger since the corresponding percentages are $45 \%$ and $65 \%$. On the contrary, low-skilled individuals seem less favorable to immigration in countries where the highly skilled are not a majority (29\%) that where they are a majority $(36 \%){ }^{7}$ With respect to the attitude toward public spending the evidence is in line with our theoretical predictions for the three groups. Among the low-skilled, we find that they are more willing to support an increase in spending if they live in a high-skilled country (43\%) than if they live in a country where the highly skilled are not a majority (36\%). For the middle-skilled, the corresponding numbers are $34 \%$ and $30 \%$, while for the highly skilled they are $36 \%$ and $34 \% .^{8}$

Ortega (2010) develops a theoretical model to analyze the political sustainability of the welfare state. In his model agents choose redistribution and immigration policy by majority vote. He shows that unskilled voters use unskilled immigration policy as a device that allows

\footnotetext{
${ }^{6}$ Regions are defined at the NUTS-1 level and the dummy variables aim to control for the average differences across regions in any unobservable characteristics.

${ }^{7}$ Differences are statistically significant for the three groups, at least at the $1 \%$ level of significance.

${ }^{8}$ Regarding opinions about public spending, differences are statistically significant for low- and middleskilled groups ( $\mathrm{p}$-value $<0.01$ in both cases). For the high-skilled, the difference is not statistically significant ( $\mathrm{p}$-value is 0.273 ).
} 
them to guarantee a higher degree of redistribution in the future. Llavador and SolanoGarcía (2011) present a political economic model in which labor-market concerns together with non-economic factors, e.g., cultural and security concerns, shape native attitudes toward immigrants. ${ }^{9}$ Our paper expands this literature by looking at the integrated predictions of both the size of the welfare state and the size of the immigration quota. Even though in our theoretical model we do not consider non-economic factors, this dimension can easily be incorporated. In that case voters would be characterized by two non-economic components: their "intrinsic" attitude toward immigrants and their political preferences. Since, by assumption, these dimensions are orthogonal to economic policy platforms, political candidates in equilibrium would still try to satisfy groups with a higher number of swing voters. ${ }^{10}$

Our paper also relates to the extensive empirical literature on immigration. The works of Dustman and Preston $(2006,2007)$ and Facchini and Mayda (2009) are of particular interest. ${ }^{11}$ Dustman and Preston (2007) estimate an structural model which considers three different channels that may shape individuals attitudes toward immigration, labor market and welfare state concerns, and cultural and racial prejudices. Regarding the second channel, they implicitly assume that the welfare state adjusts to immigration through changes in the tax rate keeping per capita benefits constant. They find that for British highly-educated people, welfare concern is the most important channel through which their preferences regarding further immigration seem to be shaped. They also find that racial factors play an important role among the lower educated. Facchini and Mayda (2009) develop and test a theoretical model in which labor market and welfare state considerations interact with each other to form public opinion about immigration. They consider a fixed exogenously given welfare system which automatically adjusts to the arrival of immigrants either by increasing the tax rate to keep per capita expenditure constant or by reducing per capita benefits, keeping the tax-rate constant. They show that attitudes toward immigration are heterogeneous

\footnotetext{
${ }^{9}$ Benhabib (1996) and Roemer and Van der Straeten (2006) also apply a political economic approach to study how immigration policies are determined. Benhabib develops a model in which the supply of immigrants is fixed and immigrants are heterogeneous in capital endowment. Roemer and Van der Straeten consider a model where voters' preferences regarding immigration and economic policies are exogenous.

${ }^{10}$ In our empirical part, though, we control for non-economic factor as drivers of opinions.

${ }^{11}$ See also Scheve and Slaughter (2001), Mayda (2006), Hanson et al. (2009) and Ortega and Polavieja (2012).
} 
across native population and that they depend on how the welfare system adjusts to the rise of the population size.

Our theoretical model shows that the arrival of immigrants first causes distributional effects (the labor market hypotheses) and then changes native preferences regarding taxexpenditure policies, suggesting that determinants of public opinion about immigration and the welfare system should be jointly estimated. One downside of the previous empirical literature is that the joint determination of preferences regarding taxes and immigration policies is typically ignored. Hence existing estimations may not capture the full extent of the effect of the welfare state channel on individual attitudes toward immigrants and the reported estimations can be biased. The current paper contributes to the empirical literature in two respects. First, our empirical illustration takes explicitly into account that preferences regarding tax-expenditure policy and immigration policy are jointly determined. Thus we provide more precise estimations of the effects that the labor market and the welfare state channels have on native attitudes toward immigration. Second, we provide empirical evidence for the European countries covered in the ESS.

The rest of the paper is organized as follow: Section 2 describes the economic environment and the political process. In Section 3 we characterize individual private decisions and Section 4 looks for political equilibrium outcomes. In Section 5, we present our empirical illustration. Section 6 concludes.

\section{The model}

\subsection{Population and technology}

Total working age population is composed of three groups: highly skilled individuals, i.e., those with a college degree, medium-skilled workers, i.e., those who completed secondary education but did not attend college, and low-skilled workers, who are those who dropped out from high school. ${ }^{12}$ Let $N_{J}$ represent the number of type $J$ workers, with $J=H, M, L$.

\footnotetext{
${ }^{12}$ We choose a model with three types of individuals instead of a simpler model with two types for two reasons. First, a theoretical model with two types of individuals (the poor and the rich) implicitly assumes that the interests of the poor are aligned with those of the middle-class, which is not necessarily true as we
} 
The total number of natives is fixed and it is equal to $N=N_{H}+N_{M}+N_{L}$. Later on, natives will decide how many (low-skilled) immigrants are allowed in. In most developed countries, the arrival of immigrants with low skills is an issue in the sense that there is a heated debate over the social and economic effects that this type of immigration can have. In this paper, we abstract from high skilled immigration since we want to study to what extent the perceptions of these effects drive natives' attitudes toward low-skilled immigrants. ${ }^{13} \mathrm{We}$ call $I$ the number of immigrants that are allowed to enter the country, with $I \in[0, \bar{I}]$. Total population will be, therefore, $P=N+I$.

There are three production factors, corresponding to the three types of labor, and everybody works the same fixed number of hours during their lifetime. Labor supply is denoted by $L_{H}, L_{M}$, and $L_{L}$ for high-, medium-, and low-skilled workers, respectively. Following Johnson (1984) we assume a linear homogeneous production function of the three types of labor $y=F\left(L_{H}, L_{M}, L_{L}\right)$, where $\frac{\partial F}{\partial L_{J}}>0$ and $\frac{\partial^{2} F}{\partial L_{J}^{2}}<0$ for $J=H, M, L$. Markets are assumed to be competitive and, therefore, the equilibrium wage of factor $J$ is $w_{J}=\frac{\partial F}{\partial L_{J}}$. By homogeneity of $F$, total labor income $\sum_{J=H, M, L} w_{J} L_{J}$, equals total output $y$. Highly skilled workers are complementary to both medium- and low-skilled workers, while medium- and low-skilled workers are perfect substitutes of each other. We also assume that the former are more productive than the latter because of their higher level of education. We rewrite the production function as $y=f\left(L_{H}, G\right)$, where $G=b L_{M}+L_{L}$, and $b \geq 1$. Then, we have $w_{M}=b w_{L}$.

Our technology implies that a rise in the number of low-skilled workers due, for example, to the inflow of immigrants, leads to a rise in the wage of highly skilled workers and to a fall in the wages of both medium- and low-skilled workers. ${ }^{14}$ Note finally that the arrival

will show in Section 4. Second, in our empirical exercise we would have to assume that parameters capturing individuals' attitudes toward policy issues do not vary between individuals who drop out from high school (the poor) and individuals who completed secondary education (the middle-class), even though they may have different interests.

${ }^{13}$ In an extension of the model we study the problem of deciding on the skill composition of an immigration flow. See Appendix 2.

${ }^{14}$ There is some controversy in the literature on whether immigrants and natives in the same skill category are perfect substitutes or not. Aydemir and Borjas (2007) and Borjas et al. $(2008,2010)$ claim that they are perfect substitutes. Card (2009), Ottaviano and Peri (2012), D'Amuri et al. (2010), and Manacorda et al. (2012) argue that they are not perfect substitutes, although they obtain very large elasticities. In contrast, Borjas, Grogger, and Hanson (2012), using the same database as Ottaviano and Peri (2012) conclude that the two groups are perfect substitutes. See also Bratsberg et al. (2014) for an overview on the literature and 
of immigrants is always positive for the country as a whole, since total output $y$ increases monotonically with $L_{L}$.

\subsection{Policy instruments and individual preferences}

The government collects tax revenue $R=\tau y$, where $\tau$ is the tax rate. Taxes are used to finance public services, which are produced using a numeraire commodity with a constant returns to scale technology. One unit of the publicly provided service is produced by using $p$ units of the numeraire. To save notation, we normalize $p=1$. Here we will refer to public services as health services, although the analysis applies also to other goods or services that can be supplemented with private purchases such as education, law enforcement, transportation, etc. $^{15}$

Individuals have preferences represented by the utility function $U(c, h)$, where $h$ represents health services and $c$ is a composite good, to which we will refer as consumption. We assume that $U(c, h)$ is quasi-concave and twice continuously differentiable over $(c, h)$. In addition, we make the standard assumptions that both $c$ and $h$ are normal goods and that the utility function is such that $\lim _{c \rightarrow 0} U(c, h)=-\infty$ and $\lim _{h \rightarrow 0} U(c, h)=-\infty$. This guarantees that individuals prefer any pair $(c, h)>>0$ to both $(c, 0)$ or $(0, h)$.

Government provides health services with quality $q$, the same for all individuals. In particular we propose $q=R / P^{\epsilon}$, where $P=N+I$ is total population, and $\epsilon \geq 0$ is a parameter that captures congestion effects. This parametrization was used first by Borcherding and Deacon (1972) and Bergstrom and Goodman (1973). ${ }^{16}$ The case $\epsilon=0$ corresponds to a pure public good where quality is completely independent of population and coincides with expenditure $R$. When $\epsilon=1$, quality is exactly per capita expenditure. The higher the parameter $\epsilon$ is, the more congested is the public good. We will focus on the case in which $\epsilon>0$, excluding the case of a pure public good.

Individuals can supplement public health services by purchasing additional health services

\footnotetext{
additional evidence with Norwegian data.

${ }^{15}$ Our model relates to the works of Coen-Pirani (2011) and Tanaka et al. (2014) that apply a political economy approach to study the effects of immigration on public spending on basic education. Considering an opting-out version of school-choice model, they find a negative relationship between these variables.

${ }^{16}$ See also Reiter and Weichenrieder (1999).
} 
in the private market. We call $s$ the amount of health services privately purchased. The cost of one unit of private health service is one unit of the numeraire. That is, we assume that there are no differences in productivity between the public and the private sector, and also that markets are competitive in the sense that private health suppliers are price-takers. The total amount of health services consumed by an individual of type $J$ is, therefore, $h_{J}=q+s_{J}$. The first part $(q)$ is common to all individuals and the second part $\left(s_{J}\right)$ is group-specific.

\subsection{Individual voting behavior}

Natives vote on candidates (parties), $A$ and $B$, whose electoral platforms specify values for the policy instruments. Let $e_{k}$ represent the policy platform of candidate $k$, with $k=$ $A, B$. In particular, $e_{k}=\left(\tau_{k}, q_{k}, I_{k}\right)$. The first and second components are the economic policy instruments and the third component describes the immigration policy. Since $q_{k}$ is completely determined by $\tau_{k}$ and $I_{k}$, we write $e_{k}=\left(\tau_{k}, I_{k}\right)$. Candidates can make binding promises on both policy instruments. Following Lindbeck and Weibull (1987), we assume there is another dimension that is relevant for voters in which candidates cannot make binding promises. Some authors call this additional dimension ideology, but this is just one possible interpretation. This ideological dimension is orthogonal to the policy platform $e_{k}$ and cannot be modified during the electoral campaign. In addition, we assume that voters within skill groups differ in their evaluation of this ideology dimension. Then, the policy platform together with candidate ideology determines voter decisions.

Consider an individual $i$ with skill level $J$. Let $W_{J}(e)$ denote $i$ 's indirect utility function, which we will explain in detail in Section 4 . As a voter, $i$ prefers candidate $A$ if:

$$
W_{J}\left(e_{A}\right)>W_{J}\left(e_{B}\right)+\sigma_{i, J}+\delta
$$

where $\sigma_{i, J}$ is an individual parameter that measures voter $i$ ideological bias toward candidate $B$. When $\sigma_{i, J}=0$, individual $i$ only cares about economic policy. When $\sigma_{i, J}>0$, she has a bias in favor of candidate $B$. We assume that $\sigma_{i, J}$ follows a uniform distribution on $\left[-\frac{1}{2 \phi^{J}}, \frac{1}{2 \phi^{J}}\right]$, with density $\phi^{J}$. On the other hand, the parameter $\delta$ measures the average 
popularity of candidate $B$ in the whole population. This parameter also follows a uniform distribution on $\left[-\frac{1}{2 \varphi}, \frac{1}{2 \varphi}\right]$, with density $\varphi$.

Parameter $\phi^{J}$ measures how sensitive individuals in group $J$ are to economic policy. When it is high, they are more concerned about economic and immigration policy than about ideological issues.

\subsection{Timing of the model}

The timing of events is as follows: (1) foresighted individuals decide the amount of private health care supplement $s$ and the amount of private consumption $c$, taken as given a policy vector $e=(\tau, I)$. (2) The two candidates announce their policy platforms simultaneously: $e_{A}$ and $e_{B}$. At this stage, both candidates are acquainted with voter policy preferences, the distributions of $\sigma_{i, J}$, and $\delta$, but not yet their realized values. (3) The value of the parameter $\delta$ is revealed. (4) The election takes place, and the elected candidate implements her announced policy platform.

\section{Individual private choices}

In Stage 1 individuals choose $s$, taking into account their expectations regarding the equilibrium policy vector $e=(\tau, I)$. The utility of a type- $J$ individual is $U\left(c_{J}, h_{J}\right)$, where $c_{J}=(1-\tau) w_{J}(I)-s_{J}, h_{J}=q+s_{J}$, and $h_{J} \geq q$. The necessary and sufficient condition that determines $s_{J}$ is:

$$
\operatorname{MRS}\left(c_{J}, h_{J}\right)=-U_{c}\left(c_{J}, h_{J}\right) / U_{h}\left(c_{J}, h_{J}\right) \leq-1 \quad\left(=-1 \text { if } s_{J}>0\right) .
$$

Figure 1 illustrates an example of the optimal choice of the rich and the poor type, respectively. Budget sets are represented with bold lines. The slope of the budget lines is -1 . In this example we assume homothetic preferences and, therefore, the slope of indifference curves is constant along the ray denoted by $H$. The rich type chooses point $\mathrm{R}$, where the condition holds with equality, $M R S\left(c_{H}, h_{H}\right)=-1$. They choose to purchase health services $s_{H}>0$ in the private market. 
Regarding the poor, if possible they would like to choose point $U$, with $s_{L}<0$. Being this unfeasible, they choose point $P$, a corner solution, where $s_{L}=0$. At the optimal solution $(P)$ the MRS is steeper than at point $U, M R S\left(c_{L}, h_{L}\right)<-1$.

Figure 1

Let $h_{J}\left((1-\tau) w_{J}(I), q\right)$ denote the demand function for health care services of an individual of type $J$, when the government provides quality $q .{ }^{17}$ Recall that, for fixed values of $\tau$ and $I$, the value of $q$ is fixed as well. Now, for a given value of $I$, since quality $q$ is an strictly increasing function of $\tau$, for each group $J$ we can find a threshold value $\widehat{\tau}_{J}$ such that $s_{J}>0$ when $\tau<\widehat{\tau}_{J}$, while $s_{J}=0$ when $\tau \geq \widehat{\tau}_{J}$. This threshold value $\widehat{\tau}_{J}$ is the one that solves $h_{J}\left(\left(1-\widehat{\tau}_{J}\right) w_{J}(I), q\left(\widehat{\tau}_{J}\right)\right)=q\left(\widehat{\tau}_{J}\right)$. The fact that both $c$ and $h$ are normal goods implies that $\widehat{\tau}_{L} \leq \widehat{\tau}_{M} \leq \widehat{\tau}_{H}$. Another implication of normality is that, for any given value of $\tau$, we get $s_{L} \leq s_{M} \leq s_{H}$

For a given value of $I$, there are three possible regimes according to the value of $\tau$ : i) Government-only provision $(G O)$ corresponds to $\tau \geq \widehat{\tau}_{H}$, since in this case the quality $q$ provided by the government is so high that even the rich do not supplement in the private market $\left(s_{L}=s_{M}=s_{H}=0\right)$; ii) All-Market $(A M)$ is the case when $\tau \leq \widehat{\tau}_{L}$, and quality $q$ is so low that the three groups decide to supplement in the private market $\left(s_{L}>0\right)$; iii) Mixed system $(G M)$, when $\tau \in\left(\widehat{\tau}_{L}, \widehat{\tau}_{H}\right)$ the poor use only public health services, i.e., $s_{L}=0$, and the rich always supplement with private purchases, $s_{H}>0$. This third case corresponds to the situation depicted in Figure 1.

\section{Political equilibrium and comparative static analysis}

We start by deriving individual preferences regarding policy platforms. Next, we characterize the political equilibrium outcome. Finally, we derive some comparative statics results with respect to individual attitudes toward economic and immigration policies.

\footnotetext{
${ }^{17}$ This is similar to Epple and Romano (1996).
} 


\subsection{Individual preferences regarding policy instruments}

To formally study candidate policy announcements at Stage 2, we first compute individual preferences regarding policy platforms. Consider a type- $J$ individual. Let $e_{J}=\left(\tau_{J}, I_{J}\right)$ denote her bliss point, which is the solution of $\left.W_{J}(e)=\max _{\{\tau, I\}}\left\{U\left((1-\tau) w_{J}(I)-s_{J}, q+s_{J}\right)\right)\right\}$, subject to the restrictions that $0 \leq \tau \leq 1$ and $0 \leq I \leq \bar{I}$. Since the utility function is continuous and the parameter space is a compact set, there is always a solution to this maximization problem. In addition, let $\zeta_{J}(\tau)$ and $\eta_{J}(I)$ denote individual $J$ 's marginal utilities. Then, the first-order conditions are:

$$
\begin{aligned}
& \zeta_{J}(\tau)=\operatorname{MRS}\left(c_{J}, h_{J}\right) w_{J}(I)+\frac{\partial q}{\partial \tau} \leq 0, \quad(=0 \text { if } \tau>0), \\
& \eta_{J}(I)=-M R S\left(c_{J}, h_{J}\right)(1-\tau) \frac{\partial w_{J}}{\partial I}+\frac{\partial q}{\partial I} \lesseqgtr 0, \quad(=0 \text { if } I \in(0, \bar{I})) .
\end{aligned}
$$

We begin by studying Equation (3), where we take $I$ as given. We can rule out a corner solution with $\tau=1$ by our assumptions on the utility function. A tax increase reduces individual $J$ 's disposable income, which entails a utility loss. The first term in Equation (3) captures this marginal cost. On the other hand, the tax increase allows for a higher provision of the public service, which in turns increases utility. The second term in Equation (3) captures this marginal benefit.

Let $\tau_{J}$ denote the optimal choice of group $J$. The following proposition ranks the preferred tax rates. The crucial parameter turns out to be $\epsilon$, the one that measures congestion in the public good. The higher is $\epsilon$, the more expensive health public provision will be, and hence the more likely that all individuals will want to supplement. This corresponds to the All-market regime (AM). Here, we focus on a configuration in which the degree of congestion is intermediate.

Proposition 1 (Ranking of tax rates) We denote by $U 0_{J}$ the indirect utility function subject to the constraint that $s_{J}=0$. Suppose that $w_{M}<y / P^{\epsilon}<w_{H}$ and assume that the slope of the function $U 0_{J}=\max _{\{\tau, q\}}\left\{U\left((1-\tau) w_{J}, q\right)\right\}$ in the space $(q, \tau)$ is lower for group $M$ than for group L. Then, $\tau_{H}=0<\tau_{L}<\tau_{M}<1$, with $\tau_{L} \in\left[\hat{\tau}_{L}, 1\right)$ and $\tau_{M} \in\left[\hat{\tau}_{M}, 1\right)$.

Proof. See Appendix 1 
The condition on the slope of the function $U 0_{J}$ is called "Slope Rising in Income (SRI)" in Epple and Romano (1996). ${ }^{18}$ This condition says that, for a given increase in quality $q$, group $M$ is willing to accept a higher increase in the tax rate than group $L$. According to Epple and Romano, empirical evidence seems to support SRI for health services. ${ }^{19}$

If $w_{L}<y / P^{\epsilon}<w_{M}$, the only change with respect to Proposition 1 is that now $\tau_{M}=0$. Note also that the condition in Proposition 1 will hold whenever $\epsilon$ is close to one, provided that the median wage is $w_{M}$. The reason is that, when $\epsilon=1, y / P^{\epsilon}$ is just mean income. In most empirical wage distributions, the median wage is lower than the mean wage. Then, we have exactly the configuration in the proposition.

We next use Expression (4) to discuss the different effects of the number of immigrants $I$ on utility. A marginal increase in the number of low-skilled immigrants affects type- $J$ 's welfare through two different channels: $(i)$ the labor market, and $(i i)$ the quality of the public service. The labor market channel is captured by the term $(1-\tau) \frac{\partial w_{J}}{\partial I}$, which is positive for highly skilled individuals, and negative for both medium- and low-skilled individuals.

With respect to the quality of the public service, the arrival of low-skilled immigrants affects the quality of public health services by an amount $\frac{\partial q}{\partial I}$, which is common for all three groups and it is equal to:

$$
\frac{\partial q}{\partial I}=\tau\left(\frac{\partial y(I)}{\partial I} \frac{1}{P^{\epsilon}}-\epsilon \frac{y(I)}{P^{1+\epsilon}}\right)
$$

The first term reflects the fact that the arrival of immigrants increases the number of taxpayers and, therefore, tax revenue goes up. However, the arrival of immigrants increases the size of the population, and hence the number of users of the public service. Therefore, for a given amount of public expenditure, the inflow of immigrants reduces $q$ by an amount $\epsilon \frac{\tau y}{P^{1+\epsilon}}$. This is the congestion effect. The final effect on $q$ will depend on which one of these two effects prevails. The resulting net effect is what we call net-tax-base effect. ${ }^{20}$

\footnotetext{
${ }^{18}$ The difference with our case is that they define preferences on $(\tau, q)$, rather than in $(q, \tau)$.

${ }^{19}$ Suppose the utility function has a CES form as $U\left(c_{J}, h_{J}\right)=\left(a\left(c_{J}\right)^{r}+(1-a)\left(h_{J}\right)^{r}\right)^{1 / r}$, with $r \leq 1$. Then SRI holds if and only if $r<0$, that is, when the two goods are complements. When $r>0$, the two goods are substitutes and we get $\tau_{M}<\tau_{L}$. Finally, when $r=0$ (Cobb-Douglas case) both groups choose exactly the same tax rate, $\tau_{L}=\tau_{M}$.

${ }^{20}$ If we define as $\mu_{I}=\frac{\partial y}{\partial I} \frac{N_{L}+I}{y}$ the output elasticity of $I$, we see that $\frac{\partial q}{\partial I} \leq 0$ if and only if $\epsilon \geq \mu_{I} \frac{P}{N_{L}+I}$. That is, when congestion effects are strong enough, an increase in the number of immigrants will always be detrimental for the quality of public health services $q$.
} 
The following proposition ranks individual preferences regarding immigration policies according to their types.

Proposition 2 (Preferences on immigration policy) Suppose that $w_{M}<y / P^{\epsilon}<w_{H}$. Then, optimal values of I for the three groups satisfy $0 \leq I_{L}=I_{M}<I_{H} \leq \bar{I}$.

Proof. See Appendix 1

\subsection{Political equilibrium outcome}

We now characterize economic and immigration policies that arise as equilibrium in the political process. Individuals vote for those policy platforms that are as close as possible to their bliss point. To study candidate decisions we have to identify the swing voter in each group $J$. This is the individual who is exactly indifferent between the platforms of candidates $A$ and $B$, i.e.:

$$
\sigma_{J}=W_{J}\left(e_{A}\right)-W_{J}\left(e_{B}\right)-\delta
$$

Expression (6) implies that those individuals in group $J$ with $\sigma_{i, J} \leq \sigma_{J}$ will vote for candidate $A$. Up to this point $\delta$ is still unknown and, therefore, the "identity" of the swing voters, and hence, the electoral outcome are random variables related to the realization of $\delta$.

Candidates $A$ and $B$ commit to policies $e_{A}$ and $e_{B}$ so as to maximize their probabilities of winning, $\pi_{A}$ and $\pi_{B}=1-\pi_{A}$, respectively. Because the candidates face the same optimization problem, and $\pi_{A}$ and $\pi_{B}$ are smooth functions of the policy platforms (see Proof of Proposition 3 below), there exists a Nash equilibrium that has both $A$ and $B$ converging to the same policy announcement: $e_{A}=e_{B}=e^{\star} \cdot{ }^{21}$ Hereafter, $e^{*}$ denotes the policy platform that arises as a political equilibrium outcome.

Proposition 3 (Political Equilibrium) Suppose that $w_{M}<y / P^{\epsilon}<w_{H}$ and that SRI holds. Then, at the political equilibrium outcome $\left(\tau^{*}, I^{*}\right)$ we have that: $0=\tau_{H} \leq \tau^{*} \leq \tau_{M}<$ 1 and $0 \leq I_{L}=I_{M} \leq I^{*} \leq I_{H} \leq \bar{I}$.

Proof. See Appendix 1

\footnotetext{
${ }^{21}$ See Lindbeck and Weibull (1987) and Persson and Tabellini (2000) for a detailed discussion of this voting model.
} 


\subsection{Comparative statics analysis}

Turnout rates vary across socioeconomic groups. Differences in these rates may make some groups more attractive in terms of votes than others, and hence more influential. The parameters $\phi^{J}$ 's capture these differences across groups $H, M$ and $L$. Precisely, we want to study what is the effect on policy announcements when the highly skilled group becomes more influential, and its consequences on low- and medium-skilled groups' attitudes toward immigration and economic policies. In terms of our model, this amounts to study what happens if the parameter $\phi^{H}$ increases (keeping $\phi^{M}$ and $\phi^{L}$ fixed).

Assumption 1 Candidate $A$ 's probability of winning is such that $\frac{\partial^{2} \pi_{A}}{\partial \tau \partial I} \leq 0$.

The cross derivative $\frac{\partial^{2} \pi_{A}}{\partial \tau \partial I}$ describes to what extent tax-expenditure and immigration policies are jointly determined. The higher its absolute value is, the stronger is the link between $\tau$ and $I$. In particular, when $\frac{\partial^{2} \pi_{A}}{\partial \tau \partial I}=0$ the equilibrium level $\tau^{*}$ does not depend on the immigration quota $I$. This is implicitly assumed by most of the existing empirical literature. ${ }^{22}$ The fact that $\frac{\partial^{2} \pi_{A}}{\partial \tau \partial I} \leq 0$ means that the marginal effect of $\tau$ on $\pi_{A}$ decreases with I. The intuition is the following. Consider the case in which $\frac{\partial \pi_{A}}{\partial \tau}$ is positive. An increase in $\tau$, all other things being constant, increases the quality of the public health service. Candidate $A$ gets some additional votes from those who are willing to support a marginal tax increase, hence $\pi_{A}$ increases. Assumption 1 says that, when the immigration quota $I$ is high, the congestion effect gets worse decreasing $q$, which reduces the marginal increase in the number of votes.

Proposition 4 Suppose that Assumption 1 holds. Then, an increase in $\phi^{H}$, ceteris paribus, decreases $\tau$ and increases $I$ at the equilibrium.

Proof. See Appendix 1

This proposition shows that policy instruments $\tau$ and $I$ are, respectively, non-increasing and non-decreasing functions of the parameter $\phi^{H}$. This is because as $\phi^{H}$ rises, the skilled

\footnotetext{
${ }^{22}$ Preferences represented by a Cobb-Douglas utility function correspond to this case.
} 
population becomes more profitable in terms of votes, and politicians tilt their announcements toward highly skilled individuals' bliss point, making the low- and medium-skilled individuals less tolerant to the arrival of immigrants.

Corollary 1 Suppose that $w_{M}<y / P^{\epsilon}<w_{H}$ and that SRI holds. If $\phi^{H}$ increases, both the low- and the medium-skilled individuals are less tolerant to the arrival of immigrants. Moreover, the medium-skilled are also more willing to rise tax-expenditure policies.

The effect of an increase in $\phi^{H}$ on the tax-expenditure preferences of the low-skilled is ambiguous. We know that at the equilibrium, $\tau^{*}$ is between $\tau_{H}$ and $\tau_{M}$. As $\phi^{H}$ increases, $\tau^{*}$ moves towards $\tau_{H}$. But, under SRI we know that $\tau_{L}$ is between $\tau_{H}$ and $\tau_{M}$ as well (see Proposition 1). So, it could be that now $\tau^{*}$ gets closer to the bliss point of the low-skilled. Corollary 1 also describes what happens with the rich group's attitude when her political influence shrinks. In that case, political competition leads to a rise in the tax rate and to a tighter immigration policy, making the rich more willing to reduce public expenditure and more favorable to increasing the immigration quota.

\section{Empirical Illustration}

We derive some testable implications from our theoretical model. First, in Section 4.1 we obtained the preferred policies for the three skill groups. In particular, propositions 1 and 2 provide a partial characterization of policy preferences regarding immigration. Second, we obtain an equilibrium configuration in Proposition 3. However, we cannot test these results without precise data on the implemented policies. Fortunately, we can do so indirectly by using the results of Corollary 1. This is what we explore next.

\subsection{Data and descriptive results}

We use data from the 4th wave (2008) of the European Social Survey (ESS). The ESS is a social survey that gathers information on attitudes and beliefs of individuals from several European countries. ${ }^{23}$ In the 2008 wave, 29 countries were covered with a total of 56,752

\footnotetext{
${ }^{23}$ See http://www.europeansocialsurvey.org/
} 
observations. ${ }^{24}$ Since we are interested in studying individual opinions about immigration we exclude four countries that have a fraction of foreign population below $1 \%{ }^{25}$ We eliminate individuals aged under 18 or over 100 (1,736 observations), and a few individuals who do not report their educational level (92 observations). We finally drop all individuals who are not citizens of the country in which they live (2,059 observations), since we are interested in the opinions of those who can vote. This reduces our sample to 46,870 individuals from 26 countries.

\subsubsection{Measuring Attitudes toward Immigrants}

There are several questions about immigration in the ESS. In particular, participants are asked their opinion about immigrants of the same ethnic group as the majority in the host country, about immigrants of a different ethnic group, and about immigrants from poorer countries outside Europe. For instance, one of questions is (variable impcntr):

To what extent (country) should allow people from the poorer countries outside Europe to come and live here?

The four possible answers are: allow many to come and live here (1), allow some (2), allow a few (3), or allow none (4). The other two questions are imsmetn and imdfetn, where people answer about their willingness to allow immigrants of the same ethnic group or from different ethnic groups as the majority of the country, respectively. ${ }^{26}$ We focus on the variable impcntr since we believe it is the one that captures better opinions on low-skilled immigration. We build a dummy variable called proimm that takes value 1 when the variable impcntr takes value 1 or 2 , and it is zero otherwise. The mean of proimm is .441 (standard deviation is .497). ${ }^{27}$

Our first observation is that there is a great deal of heterogeneity across countries with

\footnotetext{
${ }^{24}$ The list of countries is: Belgium, Bulgaria, Croatia, Cyprus, Czech Republic, Denmark, Estonia, Finland, France, Germany, Greece, Hungary, Ireland, Israel, Latvia, Netherlands, Norway, Poland, Portugal, Romania, Russia, Slovakia, Slovenia, Spain, Sweden, Switzerland, Ukraine, United Kingdom, and Turkey.

${ }^{25}$ These are Bulgaria, Poland, and Romania, with a total of 5,995 observations.

${ }^{26}$ There are others questions related to immigrants' rights and the impact immigrants have on the country's economy, culture and welfare state.

${ }^{27}$ We could alternatively construct a variable that takes value 1 only when both imdfetn and impcntr take value 1 or 2 . This would be very similar to the one we use, with a mean value of .359 (standard deviation $.479)$.
} 
respect to attitudes toward immigration as measured by our variable proimm. Figure 2 plots country means of proimm. The dotted line is the overall mean. The highest value is in Sweden (87\%) and the lowest in Cyprus (7\%).

Figure 2

We next relate individual attitudes toward immigration with the economic status of individuals. We classify them into three groups, according to their educational attainment: individuals with low, middle and high education. Later, we use this classification to test the political process predictions of the model. By using the International Standard Classification of Education (ISCED), individuals are classified into five groups: (i) less than lower secondary education (ISCED 0-1); (ii) lower secondary education completed (ISCED 2); (iii) upper secondary education completed (ISCED 3); (iv) post-secondary non-tertiary education (ISCED 4); (v) tertiary education completed (ISCED 5). Relative frequencies are 15.9\%, $13.2 \%, 32.6 \%, 3.0 \%$, and $35.3 \%$. We consider group (i) as the low-skilled group, (ii) and (iii) as the medium-skilled, and groups (iv) and (v) as highly skilled. Figure 3 represents the fraction of individuals with a positive attitude by educational level. There are striking differences across education levels. The percentage of individuals who favor immigration goes from $30 \%$ among the low-skilled to $43 \%$ and $51 \%$ among the medium and the highly skilled, respectively. ${ }^{28}$

Figure 3

To test the predictions on individuals' preferences on immigration and welfare-state policies, we use data on household income, precisely the hincfel variable in the sample. Individuals are asked how they feel about household income. Possible answers are: living comfortably on present income (1); coping on present income (2); difficult on present income (3); or very difficult on present income (4). Sample frequencies are $20.72 \%, 42.89 \%, 25.48 \%$, and $10.92 \%$. We build a dummy variable, called lowincome, that takes value 1 when hincfel is either 3 or 4 . Using this variable, we find that the mean value of proimm is $35.6 \%$ for low-income

\footnotetext{
${ }^{28}$ We find a similar educational gradient if we represent the mean values of the two other questions on immigration (imsmetn and imdfetn).
} 
individuals (lowincome $=1$ ) and it is $48.7 \%$ for the rest of individuals.

Another indirect measure of income is captured by the brwmny variable. Individuals are asked whether they find difficult to borrow money to make ends meet. Possible answers are: very difficult (1); quite difficult (2); neither easy nor difficult (3); quite easy (4); very easy (5). We build a dummy variable called borrowconstrained that takes value 1 when brwmny is either 1 or 2 . The mean of proimm is $40.3 \%$ for individuals who have problems to borrow money (borrowconstrained $=1$ ) and it is $48.5 \%$ for the rest. ${ }^{29}$

\subsubsection{Measuring Attitudes toward Public Spending}

In our model voters decide also how much to spend on public goods. We focus on one question in which individuals are asked about whether government should raise or decrease taxes and spending. The exact wording of the question (ditxssp) is the following:

Many social benefits and services are paid for by taxes. If the government had to choose between increasing taxes and spending more on social benefits and services, or decreasing taxes and spending less on social benefits and services, which should they do?

Individuals have to choose a number between 0 and 10, where 0 means that government should decrease taxes a great deal and spend much less on social benefits and 10 means government should increase taxes a great deal and spend much more on social benefits and services. If we consider that those who choose a value below 5 want to reduce spending, those who choose 5 want to keep it constant, and those who choose a value above 5 want to increase spending, we find that $29.1 \%$ want to reduce spending, $38.3 \%$ prefer to keep it constant, while $32.5 \%$ want to raise it. Alternatively, we can collapse all the information again into a dummy variable, called prospending, that takes value 1 when ditxssp takes value 6 or higher. The mean of prospending is .325 (standard deviation is .468). We find that countries are more homogeneous in attitudes toward expenditure than they are toward immigration. The maximum value of prospending is in Cyprus (.53) and the minimum in Hungary (.15).

\footnotetext{
${ }^{29}$ We could have used the variable hinctnta, which corresponds to household income. However, there is a large number of missing values in that variable (11,323 out of 46,870, a 24\%). Instead, we decided to use lowincome and borrowconstrained which do not suffer from the problem of missing values and we believe are good proxies of the financial situation in the household.
} 
We find that individuals with low education are more willing to support an increase in spending. However, contrary to the opinions on immigration, we here do not find a monotonic relationship between education levels and attitudes toward increasing spending. As Figure 3 shows, the middle-educated individuals are less favorable to increasing spending than the other groups. This result is not in line with the prediction of our theoretical model. However, at this point we have not controlled for a number of observable characteristics.

\subsubsection{Interest in Politics}

We have also information about interest in politics. We want to use this information, because not all individuals are equally likely to show up at the election polls. There are two possible sources of information. First, in one question (variable polintr) individuals report how interested in politics are. Possible answers are: very interested (1), quite interested (2), hardly interested (3), and not at all interested (4). Relative frequencies are $11.01 \%, 38.07 \%$, $32.88 \%$, and $18.04 \%$. We collapse this information into a dummy variable called polint that takes value 1 when polintr is either 1 or 2 . Second, individuals are asked whether they voted or not in the last national election (variable vote). A $76.33 \%$ went to vote, a $20.37 \%$ did not and a $3.30 \%$ were not eligible to vote.

\subsection{Model Specification}

The key implication of our theoretical model is that, as the weight of the high-skilled increases, the equilibrium outcome has lower tax-expenditure policies and a higher immigration quota. As a result, middle and low-skilled natives should be less tolerant to the arrival of immigrants and more willing to raise taxes to finance public services. To check these implications we do as follows.

We compute for each country the fraction of individuals with low, middle and high education. For instance, the fraction of individuals with high education goes from $8.26 \%$ to $64.64 \%$, with a mean value of $37.64 \%$. Next we divide all countries into two groups depending on whether or not the highly skilled make up a majority among voters. This will be the case if the group of highly skilled individuals represents a higher fraction than each one of 
the other two groups. In 4 out of 26 countries the highly skilled are a majority, while in the remaining 22 countries they are not. Combining this with the information on education levels, we end up dividing our sample into six (disjoint) groups. We illustrate this process in Table 1 below:

Table 1: Sample divided into six groups

\begin{tabular}{ccccc}
\hline \hline & \multicolumn{4}{c}{ Education level } \\
High-Skilled majority & Low & Medium & High & Total \\
\cline { 2 - 4 } & $(\mathrm{I})$ & $(\mathrm{III})$ & $(\mathrm{V})$ & \\
NO & $5,631(12.0 \%)$ & $14,690(31.3 \%)$ & $7,308(15.6 \%)$ & $27,629(58.9 \%)$ \\
& $(\mathrm{II})$ & $(\mathrm{IV})$ & $(\mathrm{VI})$ & \\
YES & $1,849(3.9 \%)$ & $6,755(14.4 \%)$ & $10,636(22.7 \%)$ & $19,241(41.0 \%)$ \\
\hline & & & & \\
Total & $7,481(15.9 \%)$ & $21,445(45.7 \%)$ & $17,944(38.3 \%)$ & $46,870(100 \%)$ \\
\hline \hline
\end{tabular}

Individuals are split into six groups, labeled from Group I to Group VI. According to Table 1, the most populous group is Group III, corresponding to individuals with a medium level of education living in countries where the highly skilled are not a majority. Our approach consists of comparing the opinions on immigration and expenditure by columns. That is, conditional on having low education, is there a significant difference in opinions depending on whether or not the highly skilled are a majority? In other words, we want to compare the opinions of Group I with those of Group II, of Group III with Group IV, and of Group V with Group VI. Our model predicts that individuals in Group II should be more opposed to immigration and more favorable to raising taxes and expenditure, compared to individuals in Group I. The reason is that those in Group II live in a country where the majority are highly skilled and the implemented policy should be closer to the bliss point of this group and, therefore, more distant from the bliss point of the low and middle-skilled. The same comment applies when we compare Group IV with Group III. Accordingly, we 
should also observe that highly skilled individuals are more favorable to immigration and more opposed to increases in taxes and expenditure in countries in which they do not make up a majority (Group V), compared to those living in countries in which they are a majority (Group VI). Now the reason is that in the former countries the policy implemented should be close to the bliss point of the low and middle educated individuals. ${ }^{30}$

A possible approach could be to compute simply the average values of our two dummy variables of interest (proimm, prospending) for each one of the six groups in Table 1 to check if our theoretical predictions hold. This is clearly problematic, since country skill composition is endogenous and may be affected by a number of unobserved characteristics that are also related to attitudes towards public spending and immigration. That is, people living in countries where the high skilled are a majority may be in general different from those living in low-skilled countries. The ideal experiment should require a variable that affects country skill composition and that it is not related to unobservable characteristics and preferences. However, it is difficult to think of such variable. To partially address this problem, we estimate a bivariate Probit model in which we control for many relevant observable characteristics and we include a dummy regional variable that aims to capture unobserved heterogeneity at the level of regions. Then, using the estimated coefficients of this model, we compute mean values of our two dummy variables for each group in Table 1. To do this, we first describe the bivariate model we estimate.

Our two binary outcomes (proimm, prospending) have been already described in the previous section. The set of regressors includes variables belonging to four different channels through which attitudes toward immigrants are driven. Namely, (i) non-economic channel, (ii) labor-market channel, (iii) welfare-state channel, and (iv) political-process channel.

(i) Non-economic channel: We include a number of individual characteristics as age, gender, religion, etc. among our regressors. Appendix 3 provides details of all the variables we use. Table 2 sets out the summary statistics for all of them. These individual characteristics are

\footnotetext{
${ }^{30}$ We could have done the analysis at the regional level, since we have information on the region of residence at the NUTS-I level. However, we prefer to do the analysis at the country level because the voting jurisdiction is the country in most cases.
} 
included in our two equations.

Table 2

(ii) The labor market channel includes years of education (eduyrs), and a dummy variable (unempl) that equals 1 if the individual is unemployed.

(iii) Concerning the welfare state channel we use data on income (lowincome and borrowconstrained). We also control for individual attitudes toward distributional issues: progov (the respondent agrees that governments should reduce income differences) and socbenpoverty (the respondent agrees that social services reduce poverty) and for individual attitudes toward political institutions: trust parliament (the respondent trusts the parliament) and health efficient (the respondent agrees that the provision of health care is efficient).

(iv) Political-process channel: Since we have six groups, we need five dummy variables to compare opinions on immigration and public spending among them. We take Group I (see Table 1 above) as the reference group and we add five dummy variables to our model. The first two dummy variables are Mideduc and Higheduc and correspond to individuals with medium and higher education, respectively. The third variable is called $H$-majority country, taking value one for those countries where the highly skilled is larger than the other two groups. Finally, we include two variables that capture the interaction of H-majority country with Mideduc and Highduc, respectively.

Our theoretical model predicts that the dummy variable $H$-majority country will have a negative sign on our first outcome (attitude to immigration), and a positive sign on our second outcome (attitude to public spending).

Opinions expressed on social spending and immigration issues can be correlated to underlying values like ideology. Since in the ESS individuals are asked about their ideological position, we control for these observable characteristics by using partisan (being close to a particular political party) and lrscale (individuals have to place themselves on a left-right scale from 0 to 10). It is important to point out that we use partisan and lrscale only as control variables with no intention to give them a causal interpretation.

Finally, we also include regional dummies in all our regressions to control for regionalspecific factors not captured by the rest of regressors. In particular, they will capture regional 
effects different from the fact that there is a particular type of majority in the country. To allow for arbitrary patterns of correlation within countries, in all of our regressions robust standard errors are clustered at country level.

\subsection{Results}

Table 3 reports the estimations of a bivariate Probit model corresponding to two alternative specifications. Model 1 includes the dummy variables we have just described. In Model 2 we present results for an alternative model in which, instead of computing majorities among all individuals, we compute them among those who went to vote in the last election. In the two models we reject the null hypothesis that the two outcome variables are independent of each other. This moves in the direction that preferences regarding immigration and preferences regarding expenditure are jointly determined. In both models the estimate of rho is 0.1, and it is significantly different from zero. This indicates that unobservable factors that are positively related to the attitude to immigration are also positively related to the attitude toward public spending. A plausible explanation for this result could be the "voting for your enemy" behavior in Ortega (2010). Some pro-redistribution voters may be willing to admit unskilled immigrants since they anticipate that these immigrants will support more redistributive policies in the future, once they become new voters.

Regarding the first channel, the variables that seem to have a significant positive effect on our first endogenous variable (proimm) are parentbornout, urban, stflife, and peoplehelp. The welfare state channel is captured by the lowincome variable. Individuals who declare to face difficulties on present income (lowincome=1) are more opposed to immigration, and more favorable to raising expenditure and taxes. The variable eduyrs seems to pick up the effects of the labor market on proimm, but its effect on prospending is not significantly different from zero. In line with our theoretical model, we find that the coefficient of $H$ majority country is negative and significant in the first equation (proimm). This means that individuals living in countries where the majority are highly skilled are more opposed to immigration than those who live in countries where a different group has a majority. In the second equation (prospending), the coefficient of H-majority country is positive. This is 
again in line with our theoretical prediction. However, its effect is not statistically significant. A possible explanation could be that the variable we use does not capture all dimensions of public spending. ${ }^{31}$

\section{Table 3}

However, what is more interesting is to use the fitted bivariate Probit model to calculate mean values of our two binary outcomes corresponding to each one of the six groups in Table 1. We represent these mean values in Figure 4. Regarding opinions about immigration the predicted pattern holds for the medium and highly skilled groups and differences are statistically significant. Mean values of proimm for these two groups are $41 \%$ and $45 \%$ when the majority is highly skilled, compared to $47 \%$ and $65 \%$ when other groups have more weight. In contrast, low-skilled individuals seem more favorable to immigration when the highly skilled are a majority. ${ }^{32}$ This goes against our theoretical prediction. Regarding opinions about public expenditure, the predicted pattern holds for the three groups. However, the difference is not statistically significant for the high-skilled. ${ }^{33}$ These results are suggestive of the modelling predictions.

Figure 4

\section{Conclusions}

This paper applies a political economy approach to explain how the interplay between immigration process, labor-market concerns, and welfare-state considerations jointly determine the shape of native preferences regarding immigration and tax-expenditure policies. A key prediction of our theoretical model is that as the rich class becomes more politically influential, the resulting political equilibrium outcome has lower public spending and larger

\footnotetext{
${ }^{31}$ To assess whether our variables of interest capture the effect that works through the political process and that years of education measures labor market skills, we run two separate bivariate Probit models, one for the subsample of natives in the labor force and another one for those not in the labor force. The estimates generally confirm that our results work mainly through those individuals who are active in the labor force. These results are available upon request.

${ }^{32}$ The p-values of the Wald tests of differences are 0.0002 for the low-skilled, 0.0012 for the medium-skilled, and below 0.0001 for the high-skilled, respectively.

${ }^{33}$ The p-values of the Wald tests of differences are 0.0025 for the low-skilled, below 0.0001 for the mediumskilled, and 0.273 for the high-skilled, respectively.
} 
immigration quotas. As a consequence, the more influential the rich group is, the higher the opposition will be against immigration among low and middle-skilled natives. That is, we find more polarization of opinions on immigration in regions in which the rich group dominates.

To test these implications we use data on 26 countries from the 2008 wave of the European Social Survey. Departing from the existing literature, we estimate the (economic and noneconomic) drivers of individual preferences regarding immigration policies, taking explicitly into account the connection between welfare-state preferences and immigration preferences. We find that individual skills have a positive influence on the probability of being more favorable to immigration, while the welfare-state channel seems to reduce that probability. Our results show that, in general, welfare state and political process channels seem to have a higher impact on preferences regarding immigration, compared to labor market channel and non-economic variables.

Our main point is that the traditional variables that have been used in the literature to explain the variation in attitudes toward immigration among individuals need to be supplemented with details on the type of political majority in the region and in the country. Since different majorities yield different policies, opinions among income groups should reflect these differences in implemented policies. 


\section{References}

[1] Aydemir, A. and G. Borjas (2007): "Cross-Country Variation in the Impact of International Migration: Canada, Mexico, and the United States," Journal of the European Economic Association 5 (4), 663-708.

[2] Benhabib, J. (1996): "On the Political Economy of Immigration," European Economic Review 40(9), 1737-1743.

[3] Bergstrom, T. and R. Goodman (1973): "Private demand for public goods," American Economic Review 63, 280-296.

[4] Blau, F. and L. Kahn (2012): "Immigration and the Distribution of Incomes." NBER Working Paper 18515.

[5] Borjas, G., Grogger, J., and G. Hansson (2008): "Imperfect Substitution between Immigrants and Natives: A Reappraisal," NBER Working Paper 13887.

[6] Borjas, G. J., Grogger, J., and G. Hansson (2010): "Immigration and the Economic Status of African-American Men," Economica 77, 255-282.

[7] Borjas, G., Grogger, J., and G. Hanson (2012): "Comment: On Estimating Elasticities of Substitution," Journal of the European Economic Association 10 (1), 198-210.

[8] Borcherding, T. and R. Deacon (1972): "The demand for the services of non-federal governments," American Economic Review 62, 891-901.

[9] Bratsberg, B., Oddbjørn, R.,Røed, M., and P. Schøne (2014): "Immigration Wage Effects by Origin," The Scandinavian Journal of Economics 116 (2): 356-393.

[10] Card, D. (2009): "Immigration and Inequality," American Economic Review 99, 1-21.

[11] Coen-Pirani, D. (2011): "Immigration and Spending on Public Education: California 1970-2000," Journal of Public Economics 95 (11-12): 1386-1396.

[12] Cunningham, P. (2006): "What accounts for differences in the use of hospital emergency departments across US communities," Health Affairs 25 (2): w324-w336. 
[13] D'Amuri, F., Ottaviano, G., and G. Peri (2010): "The Labor Market Impact of Immigration in West Germany in the 1990s," European Economic Review 54, 550-570.

[14] Dustman, Ch. and I. Preston (2006): "Is Immigration Good or Bad for the Economy? Analysis of Attitudinal Responses," Research in Labor Economics 24, 3-34.

[15] Dustman, Ch. and I. Preston (2007): "Racial and Economic Factors in Attitudes to Immigration," The B.E. Journal of Economic Analysis 8 Policy: Vol. 7: Iss. 1 (Advances), Article 62.

[16] Epple, D. and R. Romano (1996): "Public Provision of Private Goods," Journal of Political Economy 104, 57-83.

[17] Facchini, G., and A. Mayda (2009): "Does the welfare state affect individual attitudes toward immigrants? Evidence across countries," Review of Economics and Statistics 91(2), 295-314.

[18] Hanson, G., Scheve, K., and M. Slaughter (2009): "Individual preferences over highskilled immigration in the USA." In Skilled immigration today: Prospects, problems, and policies (J. Bhagwati and G. Hanson, eds.), Oxford University Press.

[19] Johnson, G. (1984): "Subsidies for higher education," Journal of Labor Economics 2, 303-318.

[20] Kerr, S. P. and W. R. Kerr (2011): "Economic Impacts of Immigration: A Survey." NBER Working Paper 16736.

[21] Llavador, H and A. Solano-García (2011): "Immigration policy with partisan parties," Journal of Public Economics 95, 134-142.

[22] Lindbeck, A. and J. Weibull (1987): "Balanced-budget redistribution as the outcome of political competition," Public Choice 52, 273-297.

[23] Manacorda, M., Manning, A., and J. Wadsworth (2012): "The Impact of Immigration on the Structure of Wages: Theory and Evidence from Britain," Journal of the European Economic Association 10 (1), 120-151. 
[24] Mayda, A. (2006): "Who is against immigration? A cross-country investigation of individual attitudes toward immigrants, " Review of Economics and Statistics 88(3), 510-530.

[25] Ortega, F. (2010): "Immigration, Citizenship, and the Size of Government," The B.E. Journal of Economic Analysis \& Policy, Contributions, Art. 26.

[26] Ortega, F. and J. Polavieja (2012): "Labor-Market Exposure as a Determinant of Attitudes toward Immigration.," Labour Economics, 19, 298-311.

[27] Ottaviani, G., and G. Peri (2012): "Rethinking the Effects of Immigration on Wages," Journal of the European Economic Association 10 (1), 152-197.

[28] Persson, T. and G. Tabellini (2000): Political Economics: Explaining economic policy, The MIT Press.

[29] Reiter, M. and A. Weichenrieder (1999): "Public goods, club goods, and the measurement of crowding," Journal of Urban Economics 46, 69-79.

[30] Roemer, J., K. Van der Straeten (2006): "The political economy of xenophobia and distribution: The case of Denmark," Scandinavian Journal of Economics 108(2), 251277.

[31] Scheve, K. and M. Slaughter (2001): "Labor Market Competition and Individual Preferences Over Immigration Policy," The Review of Economics and Statistics 83, 1, 133-145.

[32] Tanaka, R., Farré, L., and Ortega, F., (2014): "Immigration, Naturalization, and the Future of Public Education," IZA Discussion Paper 8342. 


\section{Appendix 1: Proofs}

Before presenting the proof of Proposition 1, we present the following Lemma that characterizes the optimal choice of $\tau$ for any group $J$.

Lemma 1 (Preferences on tax rates): Consider group J. There are three possible cases depending on the value of $\epsilon: i)$ When $\epsilon$ is large so that $y / P^{\epsilon}<w_{J}$, the optimal tax rate for group $J$ is $\tau_{J}=0$; ii) When $\epsilon$ is low so that $y / P^{\epsilon}>w_{J}$, the optimal tax rate for group $J$ lies in the interval $\left[\widehat{\tau}_{J}, 1\right)$; iii) If $y / P^{\epsilon}=w_{J}$, any value of $\tau$ in the interval $\left[0, \widehat{\tau}_{J}\right]$ is optimal.

Proof: Case (i) is illustrated in Figure A1, top panel. We need to define an auxiliary function, $U 1_{J}$ which is the indirect utility function assuming that $s_{J}$ can take both positive and negative values. If $s_{J}$ can be negative, then $M R S\left(c_{J}, h_{J}\right)=-1$ since the first-order Condition (2) when choosing $s$ always holds with equality. The derivative of $U 1_{J}$ with respect to $\tau$ is $-w_{J}+\frac{\partial q}{\partial \tau}=-w_{J}+y / P^{\epsilon}$, which is negative. Then, in Case (i) $U 1_{J}$ is always decreasing in $\tau$. Note that the function $U 0_{J}$, in Proposition 1, is the indirect utility function but assuming that $s_{J}=0$. Because of our assumptions on the original utility function, the function $U 0_{J}$ has an inverted-U shape. It is tangent to $U 1_{J}$ exactly when $\tau=\widehat{\tau}_{J}$, because in this case the individual voluntarily chooses $s_{J}=0$. For other values of $\tau, U 0_{J}$ lies below $U 1_{J}$. The true indirect utility function coincides with $U 1_{J}$ to the left of $\widehat{\tau}_{J}$ since $s_{J}>0$ when $\tau<\widehat{\tau}_{J}$, and it coincides with $U 0_{J}$ to the right of $\widehat{\tau}_{J}$ since $s_{J}=0$ when $\tau \geq \widehat{\tau}_{J}$. It is represented in bold type in Figure A1. In this case the indirect utility function is always decreasing in $\tau$, which means that the preferred tax rate is $\tau_{J}=0$.

Case (ii) is represented in the bottom panel of Figure A1. The difference with Case (i) is that now the function $U 1_{J}$ is strictly increasing. But then, the tangency between $U 1_{J}$ and $U 0_{J}$ happens in the increasing part of $U 0_{J}$. The indirect utility function has a maximum to the right of $\widehat{\tau}_{J}$. In Figure A1 this maximum is at $\tau=\tau_{J} \in\left[\widehat{\tau}_{J}, 1\right)$.

Case (iii) is similar to cases (i) and (ii), but now the function $U 1_{J}$ is horizontal and it is tangent to $U 0_{J}$ at the point where $U 0_{J}$ reaches a maximum. Since this is precisely $\widehat{\tau}_{J}$, the indirect utility function is constant on $\left[0, \widehat{\tau}_{J}\right]$ and decreasing when $\tau>\widehat{\tau}_{J}$.

Figure A1 
Proof of Proposition 1 (Ranking of tax rates): The fact that $\tau_{H}=0$ is a direct implication of Lemma 1. To prove the second part, note that both $M$ and $L$ choose an interior solution for $\tau$. In particular, they choose the values that maximize $U 0_{M}$ and $U 0_{L}$, respectively. The first-order conditions are satisfied with equality:

$$
\begin{aligned}
\frac{\partial U 0_{M}\left((1-\tau) w_{M}, q\right)}{\partial \tau} & =-\frac{\partial U 0_{M}}{\partial c} w_{M}+\frac{\partial U 0_{M}}{\partial h} \frac{\partial q}{\partial \tau}=0 \\
\frac{\partial U 0_{L}\left((1-\tau) w_{L}, q\right)}{\partial \tau} & =-\frac{\partial U 0_{L}}{\partial c} w_{L}+\frac{\partial U 0_{L}}{\partial h} \frac{\partial q}{\partial \tau}=0 .
\end{aligned}
$$

These two equations can be written as:

$$
\begin{aligned}
\frac{\partial U 0_{M} / \partial c}{\partial U 0_{M} / \partial h} w_{M} & =\frac{\partial q}{\partial \tau} \\
\frac{\partial U 0_{L} / \partial c}{\partial U 0_{L} / \partial h} w_{L} & =\frac{\partial q}{\partial \tau}
\end{aligned}
$$

The term on the left represents the marginal cost of the tax rate, while the term on the right represents the marginal benefit. The marginal benefit does not depend on $\tau$, while the marginal cost is increasing in $\tau$. Now notice that the marginal cost is exactly the slope of the function $U 0_{J}$ in the space $(q, \tau)$. Then, $S R I$ condition implies that the marginal cost for type $M$ is below the marginal cost of type $L$, then we have that $\tau_{M}>\tau_{L}$.

Proof of Proposition 2 (Preferences on immigration policy): Since both $w_{L}$ and $w_{M}$ are less than $y / P^{\epsilon}$, we have that at their optimal choices of $\tau, s_{M}=s_{L}=0$. First-order conditions for $\tau$ are:

$$
\operatorname{MRS}\left(c_{J}, h_{J}\right)=-\frac{\partial q}{\partial \tau} / w_{J}(I)
$$

for $J=L, M, H$. First-order conditions for $I$ can be written for groups $M$ and $L$ as:

$$
\begin{aligned}
&-M R S\left(c_{M}, h_{M}\right)(1-\tau) \frac{\partial w_{M}}{\partial I}+\frac{\partial q}{\partial I} \lesseqgtr 0 \lesseqgtr \\
&-M R S\left(c_{L}, h_{L}\right)(1-\tau) \frac{\partial w_{L}}{\partial I}+\frac{\partial q}{\partial I} \lesseqgtr 0 .
\end{aligned}
$$


Substituting from the above first-order conditions for $\tau$ :

$$
\begin{aligned}
& \frac{\frac{\partial w_{M}}{\partial I}}{w_{M}(I)}(1-\tau) \frac{\partial q}{\partial \tau}+\frac{\partial q}{\partial I} \lesseqgtr 0 \lesseqgtr \\
& \frac{\frac{\partial w_{L}}{\partial I}}{w_{L}(I)}(1-\tau) \frac{\partial q}{\partial \tau}+\frac{\partial q}{\partial I} \lesseqgtr 0 .
\end{aligned}
$$

Our technology choice implies $w_{M}=b w_{L}$ and, therefore, $\frac{\partial w_{M}}{\partial I}=b \frac{\partial w_{L}}{\partial I}$. But then, the firstorder condition for both groups $(M$ and $L)$ is the same which implies $I_{L}=I_{M}$.

To prove that $I_{M}<I_{H}$ we simply compare the first-order conditions of groups $M$ and $H$. The condition for group $M$ is Equation (12) above. The one of group $H$ is:

$$
-M R S\left(c_{H}, h_{H}\right)(1-\tau) \frac{\partial w_{H}}{\partial I}+\frac{\partial q}{\partial I} \lesseqgtr 0
$$

The difference is that in Equation (16) the term on the left is positive, since $\frac{\partial w_{H}}{\partial I}>0$. This guarantees that group $H$ will maximize utility at a value of $I$ to the right of $I_{M}$. When congestion is low and $\epsilon<\mu_{I} \frac{P}{N_{L}+I}$, we have $\frac{\partial q}{\partial I}>0$ and group $H$ will want to have $I_{H}=\bar{I}$. When $\epsilon \geq \mu_{I} \frac{P}{N_{L}+I}, \frac{\partial q}{\partial I}<0$ and $I_{L}=I_{M}=0$.

Proof of Proposition 3 (Political equilibrium): We use Expression 6 to compute Candidate $A$ 's probability of winning, which is equal to

$$
\begin{aligned}
\pi_{A}\left(e_{A}, e_{B}\right) & =\operatorname{Prob}\left[\sum_{J} \int_{-\frac{1}{2 \phi^{J}}}^{\sigma^{J}} d \sigma_{i, J} \geq \frac{1}{2}\right] \\
& =\frac{1}{2}+\frac{\varphi}{\phi}\left[\sum_{J} \frac{N_{J} \phi^{J}}{N}\left(W_{J}\left(e_{A}\right)-W_{J}\left(e_{B}\right)\right)\right]
\end{aligned}
$$

where $\phi=\sum_{J} \frac{N_{J} \phi^{J}}{N}$ is the average density across groups. Candidate $B$ 's probability of winning is, therefore, equal to $1-\pi_{A}$. Note that $\pi_{A}$ is continuous in $e_{A}$.

Because both candidates face the same optimization problem, we focus on Candidate $A$. She chooses a policy platform $e_{A}$ so as to maximize $\pi_{A}\left(e_{A}, e_{B}\right)$ taken platform $e_{B}$ as given. From Expression (17) we see that Candidate $A$ is, in fact, maximizing a weighted social welfare function. This optimization problem has one interior solution and eight corner 
solutions. As the key implication of our theoretical model (Section 4.3) does not depend on the nature of the solution, we restrict our attention to the interior solution, and the first-order conditions are:

$$
\begin{aligned}
& \frac{\partial L}{\partial \tau}=\frac{\varphi}{\phi} \sum_{J} \frac{N_{J} \phi^{J}}{N} \zeta_{J}(\tau)=0 . \\
& \frac{\partial L}{\partial I}=\frac{\varphi}{\phi} \sum_{J} \frac{N_{J} \phi^{J}}{N} \eta_{J}(I)=0 .
\end{aligned}
$$

Since $\pi_{A}$ is continuous and the parameter space is a compact set, there is a policy platform $\left(\tau^{*}, I^{*}\right)$ that solves the equation system (18)-(19); and it balances the opposite effects (weighted by group relative size and group sensitiveness to policy issues) that work through the labor market and the welfare system.

From Proposition 1 we have that $\tau_{H}=0<\tau_{L}<\tau_{M}<1$. As a result, $\tau^{\star} \in\left[\tau_{H}, \tau_{M}\right]$, since it will never be optimal to choose an income tax rate above $\tau_{M}$; a similar reasoning applies to $I^{\star}$.

Proof of Proposition 4: Consider first the interior solution case. Equations (18) and (19) determine $\tau$ and $I$ as functions of the parameters $\phi^{H}, \phi^{M}$, and $\phi^{L}$. Let $H M$ be the Hessian matrix and $|H M|$ its determinant. We differentiate equations (18) and (19) to obtain the effect that a change in $\phi^{H}$ has on our policy instruments:

$$
\begin{gathered}
\frac{\partial \tau}{\partial \phi^{H}}=\frac{1}{|H M|} \frac{N_{H}}{N}\left[-\zeta_{H}(\tau) \frac{\partial^{2} \pi_{A}}{\partial I^{2}}+\eta_{H}(I) \frac{\partial^{2} \pi_{A}}{\partial I \partial \tau}\right] . \\
\frac{\partial I}{\partial \phi^{H}}=\frac{1}{|H M|} \frac{N_{H}}{N}\left[-\eta_{H}(I) \frac{\partial^{2} \pi_{A}}{\partial \tau^{2}}+\zeta_{H}(\tau) \frac{\partial^{2} \pi_{A}}{\partial I \partial \tau}\right] .
\end{gathered}
$$

Note that $|H M|$ is positive from the second-order conditions. Since $0=\tau_{H} \leq \tau^{*} \leq \tau_{M}$ and $I_{M} \leq I^{*} \leq I_{H}$ we have $\zeta_{H}(\tau) \leq 0$ and $\eta_{H}(I) \geq 0$, implying that $\frac{\partial \tau}{\partial \phi^{H}} \leq 0$ and $\frac{\partial I}{\partial \phi^{H}} \geq 0$.

If the parameters of the model are such that we have a corner solution in which either $\tau^{*}$ or $I^{*}$ is not at a corner, then an increase in $\phi^{H}$ may only affect the optimal value of the variable whose restriction is not binding. It is also possible, however, that changes in $\phi^{H}$ move the optimal solution away from the corner. For instance, consider the case where 
$\tau^{*} \in(0,1)$ and $I^{*}=0$. An increase in $\phi^{H}$ makes the highly skilled more profitable in terms of votes and, hence, candidates tilt their policy announcement toward the bliss point of the highly skilled group. This means that $\tau^{*}$ decreases and $I^{*}$ becomes strictly positive. Now, consider the case where the parameters of the model are such that the equilibrium policy is $\left(0, I^{*}\right)$. Under this scenario, a rise in $\phi^{H}$ may only affect the optimal value of $I^{*}$ since there is no room to change the value of $\tau$. An analogous argument applies to other corner solutions. 


\section{Appendix 2: Deciding the skill mix of immigrants}

Here we study the case in which the quota of immigrants $I$ is given, but natives have to choose the skill mix of this immigrants flow. In particular, they have to choose the fraction $\alpha$ of immigrants that are low-skilled, while $(1-\alpha)$ is the percentage of high-skilled immigrants. ${ }^{34}$ Now total population $P=N+I$ is fixed. Labor supplies of the three factors are $L_{H}=N_{H}+(1-\alpha) I, L_{M}=N_{M}$, and $L_{L}=N_{L}+\alpha I$, respectively. Our technology implies that an increase in $\alpha$ raises the wage of the high-skilled, while it reduces the wages of both medium- and low-skilled workers. The effect of $\alpha$ on total output is:

$$
\frac{\partial y}{\partial \alpha}=\frac{I}{P}\left\{\sum_{J}\left(\frac{\partial w_{J}}{\partial L_{L}}-\frac{\partial w_{J}}{\partial L_{H}}\right) L_{J}+\left(w_{L}-w_{H}\right)\right\} .
$$

There are two opposite effects at work. On the one hand, there is a redistribution effect (whose sign is ambiguous) which favors high-skilled workers at the expense of low- and medium-skilled workers. This effect is captured by the first term. On the other hand, the term $\left(w_{L}-w_{H}\right)$ measures the fall in the output due to the substitution between high-skilled immigrants and low-skilled immigrants. The sign of this expression is ambiguous, although we should expect it to be negative, since most countries seem to be interested in attracting high-skilled immigrants.

The effect of $\alpha$ on the quality of public services, $q$, will depend on the sign of the above derivative. If $\frac{\partial y}{\partial \alpha}<0$, then $\frac{\partial q}{\partial \alpha}<0$ as well. Individual decisions on $s$ are the same as in the Section 3. Regarding individual preferences on policy instruments, we can obtain similar results to those in Section 4. In particular, we have that assuming $w_{M}<\frac{y}{P^{\epsilon}}<w_{H}$ and SRI, we get $\tau_{H}=0<\tau_{L}<\tau_{M}<1$, and $0 \leq \alpha_{L}=\alpha_{M}<\alpha_{H} \leq 1$. The analysis of the political equilibrium outcome does not change. Recall that the resulting political equilibrium policy $\left(\tau^{\star}, \alpha^{\star}\right)$ is closer to the bliss point of that group with the highest number of swing voters. To simplify the comparative static analysis we consider the case in which the parameter space is such that $\left(\tau^{\star}, \alpha^{\star}\right)$ is an interior solution. Then, we can prove a result similar to Proposition 4. In particular, we denote by $\Pi_{\tau \alpha}$ the cross-derivative of the objective function

\footnotetext{
${ }^{34}$ To have a tractable model we do not allow for medium-skilled immigrants, reducing the problem to just one dimension.
} 
with respect to $\tau$ and $\alpha$. If $\Pi_{\tau \alpha}$ is non-positive and $w_{M}<\frac{y}{P^{\epsilon}}<w_{H}$, an increase in $\phi^{H}$, ceteris paribus, decreases $\tau$ and increases $\alpha$ at the equilibrium. Clearly, since in the new equilibrium there will be relatively more low-skilled immigrants, we should observe that lowand medium-skilled natives are less tolerant to the arrival of this type of foreign workers. 


\section{Appendix 3: Description of variables in the text}

We describe all the variables used in our estimations (names in bold type). We exclude those already discussed in the text. All variables in italics are from ESS, 2008 wave. ${ }^{35}$

- Believe people is helpful (peoplehelp): dummy variable that is 1 when $p p l h l p$ is above 5. Variable pplhlp is the answer to "Most of the time people helpful or mostly looking out for themselves." Answers go from 0 ("Most mostly look out for themselves") to 10 ("People mostly try to be helpful").

- Parent born out (parentbornout): dummy variable that is 1 when either the father (facntr is 2) or the mother (mocntr is 2) was not born in the country.

- Born out of country (bornout): dummy variable that equals 1 when the individual was not born in the country (brncntr is 2).

- Urban area (urban): dummy variable that equals 1 when the individual lives in a big city (domicil is 1 ) or in the suburbs or outskirts of a big city (domicil is 2).

- Government should reduce income differences (progov): dummy variable that equals 1 when gincdif is 1 or 2. Variable gincdif is the answer to "Government should reduce differences in income levels." Possible answers are 1 ("Agree strongly"), 2 ("Agree"), 3 ("Neither agree nor disagree"), 4 ("Disagree"), and 5 ("Disagree strongly").

- Social services reduce poverty (socbenpoverty): dummy variable that equals 1 when sbprvpv is 1 or 2 . Variable sbprvpv is the answer to "Social benefits/services prevent widespread poverty." Possible answers are 1 ("Agree strongly"), 2 ("Agree"), 3 ("Neither agree nor disagree"), 4 ("Disagree"), and 5 ("Disagree strongly").

- Trust in parliament (trustparliament): dummy variable that equals 1 when trstprl is above 4. Variable trstprl is the answer to "Trust in country's parliament." Possible answers go from 0 ("No trust at all") to 10 ("Complete trust").

\footnotetext{
${ }^{35}$ For more details, go to:

http://ess.nsd.uib.no/ess/round4/
} 
- Provision of health care efficient (healthefficient): dummy variable that equals 1 when hlthcef is above 5. Variable hlthcef is the answer to "Provision of health care, how efficient." Possible answers go from 0 ("Extremely inefficient") to 10 ("Extremely efficient").

- Unemployed last 3 months (unempl): dummy variable that equals 1 when uemp3 is 1. Variable uemp3 is 1 when the individual has been ever unemployed and seeking for a work for a period of more than three months.

- Ideological position (Irscale): categorical variable that reflects placement on left to right scale. Possible categories go from 0 (Left) to 10 (Right).

- Close to a party (partisan): dummy variable that equals 1 when clsprty is 1 . Variable clsprty is 1 when the individual declares that she feels closer to a particular political party.

- Life satisfaction (stflife): categorical variable that reflects the answer to "How satisfied with life as a whole." Possible categories go from 0 (Extremely dissatisfied) to 10 (Extremely satisfied).

- Good health (healthy): dummy variable that equals 1 when health is 1 or 2 . Variable health is the answer to "Subjective general health." Possible answers are 1 ("Very good"), 2 ("Good"), 3 ("Fair"), 4 ("Bad"), and 5 ("Very bad").

- Elementary occupation (manualworker): dummy variable that equals 1 when iscoco is greater or equal than 9,000. Variable iscoco is a 4-digit coding of occupations. 
Table 2: Summary statistics

\begin{tabular}{lccccc}
\hline \hline \multicolumn{1}{c}{ Variable } & Mean & (Std. Dev.) & Min. & Max. & N \\
\hline Pro-immigration attitude & 0.441 & $(0.497)$ & 0 & 1 & 44835 \\
Pro-spending attitude & 5.100 & $(2.148)$ & 0 & 10 & 42488 \\
prospending & 0.325 & $(0.468)$ & 0 & 1 & 42488 \\
Age & 46.795 & $(17.62)$ & 18 & 99 & 46870 \\
Age squared & 2500.199 & $(1754.395)$ & 324 & 9801 & 46870 \\
Female & 0.541 & $(0.498)$ & 0 & 1 & 46853 \\
Religious & 0.619 & $(0.486)$ & 0 & 1 & 46614 \\
Born out of country & 0.057 & $(0.232)$ & 0 & 1 & 46822 \\
Parent born out & 0.122 & $(0.328)$ & 0 & 1 & 46616 \\
Urban area & 0.376 & $(0.484)$ & 0 & 1 & 46677 \\
Life satisfaction & 6.203 & $(2.53)$ & 0 & 10 & 46478 \\
Good health & 0.562 & $(0.496)$ & 0 & 1 & 46815 \\
Believe people is helpful & 0.317 & $(0.465)$ & 0 & 1 & 46617 \\
Household income & 5.267 & $(2.735)$ & 1 & 10 & 35547 \\
Low income & 0.364 & $(0.481)$ & 0 & 1 & 46511 \\
Problems to borrow & 0.514 & $(0.5)$ & 0 & 1 & 44668 \\
Gvt should reduce income differences & 0.72 & $(0.449)$ & 0 & 1 & 46079 \\
Trust in parliament & 0.502 & $(0.5)$ & 0 & 1 & 45756 \\
Social services reduce poverty & 0.522 & $(0.5)$ & 0 & 1 & 45371 \\
Provision of health care efficient & 0.429 & $(0.495)$ & 0 & 1 & 45987 \\
Education (years) & 12.07 & $(4.238)$ & 0 & 48 & 46482 \\
Unemployed last 3 months & 0.264 & $(0.441)$ & 0 & 1 & 46567 \\
Elementary occupation & 0.107 & $(0.309)$ & 0 & 1 & 41283 \\
Low education & 0.16 & $(0.366)$ & 0 & 1 & 46870 \\
Middle education & 0.458 & $(0.498)$ & 0 & 1 & 46870 \\
High education & 0.383 & $(0.486)$ & 0 & 1 & 46870 \\
Interest in politics & 0.491 & $(0.5)$ & 0 & 1 & 46741 \\
Vote last election & 0.763 & $(0.425)$ & 0 & 1 & 46436 \\
High-skilled majority & 0.411 & $(0.492)$ & 0 & 1 & 46870 \\
High-skilled majority among voters & 0.418 & $(0.493)$ & 0 & 1 & 46870 \\
High-skilled majority among interested in politics & 0.497 & $(0.5)$ & 0 & 1 & 46870 \\
Close to a party & 0.514 & $(0.5)$ & 0 & 1 & 45750 \\
Ideological position & 5.103 & $(2.173)$ & 0 & 10 & 40204 \\
\hline
\end{tabular}




\section{TABLES AND FIGURES}

Table 3: Bivariate Probit Estimations

\begin{tabular}{|c|c|c|c|c|}
\hline \multirow[b]{2}{*}{ VARIABLES } & \multicolumn{2}{|c|}{ Model 1} & \multicolumn{2}{|c|}{ Model 2} \\
\hline & Proimm & Prospending & Proimm & Prospending \\
\hline \multicolumn{5}{|l|}{ Non-Economic Channel } \\
\hline Age & $\begin{array}{c}0.00508 \\
(0.00496)\end{array}$ & $\begin{array}{c}0.00378 \\
(0.00552)\end{array}$ & $\begin{array}{c}0.00507 \\
(0.00496)\end{array}$ & $\begin{array}{c}0.00379 \\
(0.00553)\end{array}$ \\
\hline Age squared & $\begin{array}{c}-0.0001 * * \\
(0.00005)\end{array}$ & $\begin{array}{c}-0.0000 \\
(0.00005)\end{array}$ & $\begin{array}{l}-0.0001 * * \\
(0.00005)\end{array}$ & $\begin{array}{c}-0.0000 \\
(0.00005)\end{array}$ \\
\hline Female & $\begin{array}{l}-0.00217 \\
(0.0316)\end{array}$ & $\begin{array}{c}0.0115 \\
(0.0277)\end{array}$ & $\begin{array}{l}-0.00241 \\
(0.0314)\end{array}$ & $\begin{array}{c}0.0115 \\
(0.0277)\end{array}$ \\
\hline Religious & $\begin{array}{l}-0.0358 \\
(0.0295)\end{array}$ & $\begin{array}{l}-0.0182 \\
(0.0353)\end{array}$ & $\begin{array}{l}-0.0359 \\
(0.0295)\end{array}$ & $\begin{array}{l}-0.0183 \\
(0.0353)\end{array}$ \\
\hline Born out of country & $\begin{array}{c}0.122 \\
(0.0794)\end{array}$ & $\begin{array}{c}-0.00988 \\
(0.0743)\end{array}$ & $\begin{array}{c}0.121 \\
(0.0794)\end{array}$ & $\begin{array}{r}-0.00989 \\
(0.0743)\end{array}$ \\
\hline Parent born out & $\begin{array}{l}0.156^{* *} \\
(0.0790)\end{array}$ & $\begin{array}{l}-0.0319 \\
(0.0618)\end{array}$ & $\begin{array}{c}0.155^{* *} \\
(0.0792)\end{array}$ & $\begin{array}{l}-0.0318 \\
(0.0618)\end{array}$ \\
\hline Urban area & $\begin{array}{c}0.0791 * * * \\
(0.0230)\end{array}$ & $\begin{array}{c}0.0364^{*} \\
(0.0196)\end{array}$ & $\begin{array}{c}0.0794 * * * \\
(0.0231)\end{array}$ & $\begin{array}{l}0.0365^{*} \\
(0.0196)\end{array}$ \\
\hline Life satisfaction & $\begin{array}{c}0.0244 * * \\
(0.0116)\end{array}$ & $\begin{array}{c}0.0134 * * * \\
(0.00333)\end{array}$ & $\begin{array}{c}0.0244 * * \\
(0.0116)\end{array}$ & $\begin{array}{c}0.0134 * * * \\
(0.00333)\end{array}$ \\
\hline Good health & $\begin{array}{c}0.0246 \\
(0.0358)\end{array}$ & $\begin{array}{l}-0.0225 \\
(0.0197)\end{array}$ & $\begin{array}{c}0.0249 \\
(0.0359)\end{array}$ & $\begin{array}{l}-0.0224 \\
(0.0197)\end{array}$ \\
\hline Believe people is helpful & $\begin{array}{c}0.206 * * * \\
(0.0368)\end{array}$ & $\begin{array}{c}0.126 * * * \\
(0.0326)\end{array}$ & $\begin{array}{c}0.206 * * * \\
(0.0367)\end{array}$ & $\begin{array}{c}0.126 * * * \\
(0.0326)\end{array}$ \\
\hline \multicolumn{5}{|l|}{ Welfare State Channel } \\
\hline Low income & $\begin{array}{c}-0.0785 * * * \\
(0.0278)\end{array}$ & $\begin{array}{c}0.0690 * * \\
(0.0284)\end{array}$ & $\begin{array}{c}-0.0780 * * * \\
(0.0278)\end{array}$ & $\begin{array}{c}0.0690 * * \\
(0.0284)\end{array}$ \\
\hline Problems to borrow & $\begin{array}{c}-0.0321 * * \\
(0.0127)\end{array}$ & $\begin{array}{l}0.00763 \\
(0.0304)\end{array}$ & $\begin{array}{c}-0.0319 * * \\
(0.0128)\end{array}$ & $\begin{array}{l}0.00770 \\
(0.0304)\end{array}$ \\
\hline Gvt should reduce income differences & & $\begin{array}{c}0.228 * * * \\
(0.0328)\end{array}$ & & $\begin{array}{c}0.228 * * * \\
(0.0328)\end{array}$ \\
\hline Trust in parliament & & $\begin{array}{c}0.127 * * * \\
(0.0336)\end{array}$ & & $\begin{array}{c}0.127 * * * \\
(0.0336)\end{array}$ \\
\hline Social services reduce poverty & & $\begin{array}{c}0.107 * * * \\
(0.0316)\end{array}$ & & $\begin{array}{c}0.107 * * * \\
(0.0316)\end{array}$ \\
\hline Provision of health care efficient & & $\begin{array}{c}0.211 * * * \\
(0.0259)\end{array}$ & & $\begin{array}{c}0.211 * * * \\
(0.0260)\end{array}$ \\
\hline \multicolumn{5}{|l|}{ Labor Market Channel } \\
\hline Education (years) & $\begin{array}{c}0.0254 * * * \\
(0.00740)\end{array}$ & $\begin{array}{c}0.00303 \\
(0.00497)\end{array}$ & $\begin{array}{c}0.0838 \\
(0.0735)\end{array}$ & $\begin{array}{l}-0.0304 \\
(0.0647)\end{array}$ \\
\hline Unemployed last 3 months & $\begin{array}{c}0.0208 \\
(0.0391)\end{array}$ & $\begin{array}{l}0.00423 \\
(0.0402)\end{array}$ & $\begin{array}{c}0.336 \text { *** } \\
(0.124)\end{array}$ & $\begin{array}{c}0.0854 \\
(0.0888)\end{array}$ \\
\hline \multicolumn{5}{|l|}{ Political Process Channel } \\
\hline Middle education & $\begin{array}{c}0.0812 \\
(0.0738)\end{array}$ & $\begin{array}{l}-0.0294 \\
(0.0648)\end{array}$ & $\begin{array}{c}0.132 * * * \\
(0.0437)\end{array}$ & $\begin{array}{c}0.109 * * * \\
(0.0228)\end{array}$ \\
\hline
\end{tabular}




\begin{tabular}{lcccc} 
High education & $0.338^{* * *}$ & 0.0846 & $-0.0628^{* * *}$ & $-0.0417 * *$ \\
& $(0.123)$ & $(0.0884)$ & $(0.0175)$ & $(0.0192)$ \\
H-majority country & $-0.431^{* * *}$ & 0.132 & $-0.428^{* * *}$ & 0.131 \\
& $(0.0800)$ & $(0.121)$ & $(0.0817)$ & $(0.121)$ \\
H-majority country*Middle education & 0.0168 & -0.0930 & -0.00236 & -0.0900 \\
& $(0.0694)$ & $(0.0838)$ & $(0.0704)$ & $(0.0841)$ \\
H-majority country*High education & $-0.240^{*}$ & -0.161 & $-0.238^{*}$ & -0.161 \\
& $(0.127)$ & $(0.123)$ & $(0.129)$ & $(0.122)$ \\
Close to a party & $0.132^{* * *}$ & $0.109 * * *$ & $0.112^{* *}$ & $0.102^{* * *}$ \\
& $(0.0437)$ & $(0.0228)$ & $(0.0524)$ & $(0.0281)$ \\
Ideological position & $-0.0629 * * *$ & $-0.0417 * *$ & $-0.0625^{* * *}$ & $-0.0391 * *$ \\
& $(0.0175)$ & $(0.0192)$ & $(0.0183)$ & $(0.0199)$ \\
& & & & \\
\hline Observations & & & & \\
Rho & 33,036 & 33,036 & 33,036 & 33,036 \\
& $0.100^{* * *}$ & & $0.1000^{* * *}$ & \\
\hline \hline
\end{tabular}

Notes: Robust standard errors in parentheses; *** $\mathrm{p}<0.01, * * \mathrm{p}<0.05, * \mathrm{p}<0.1$

The model includes regional dummy variables at NUTS-1 level (not reported here). Errors are clustered at the country level. 


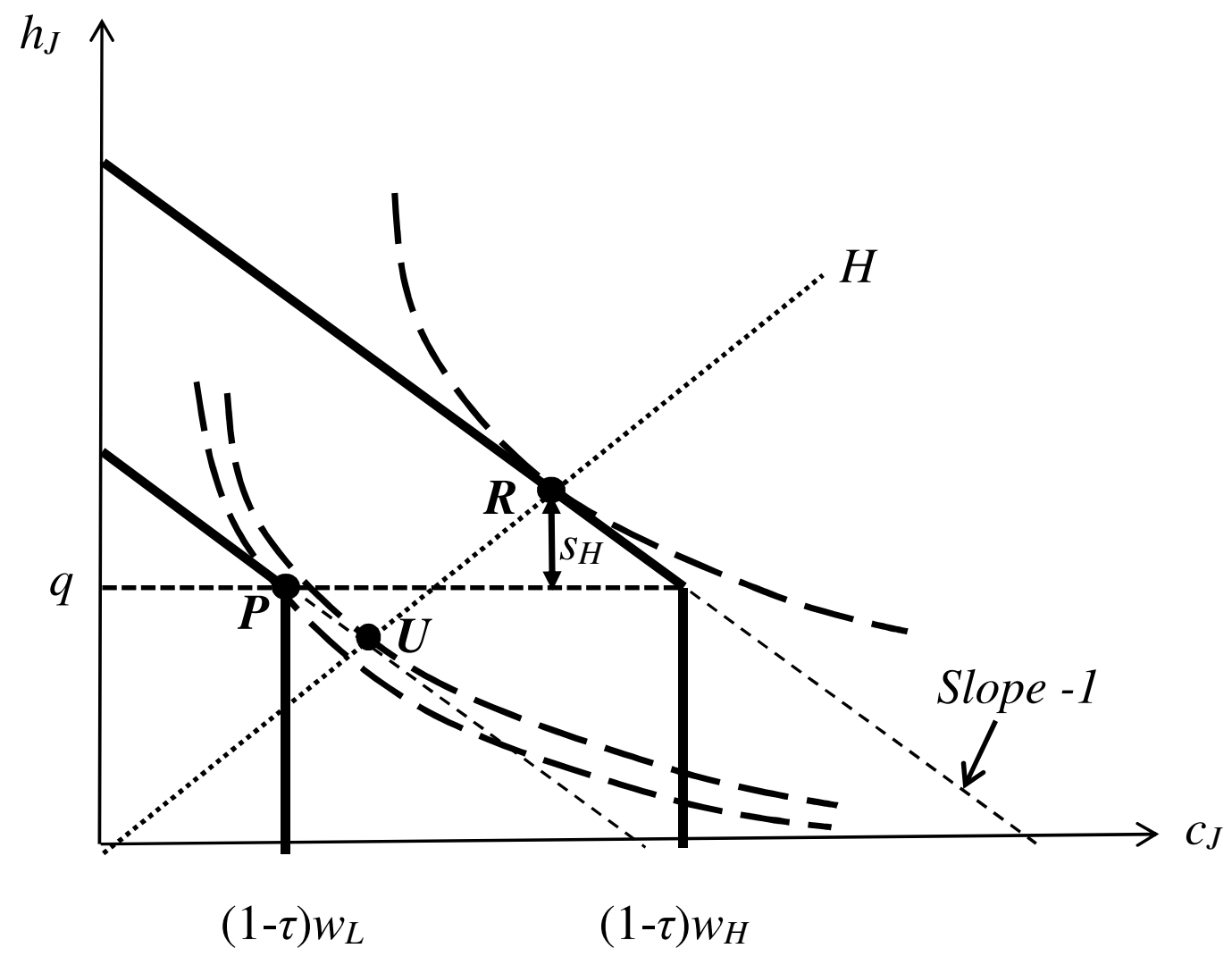

Figure 1: Individual private choices 
Figure 2: Pro-immigration attitude by country

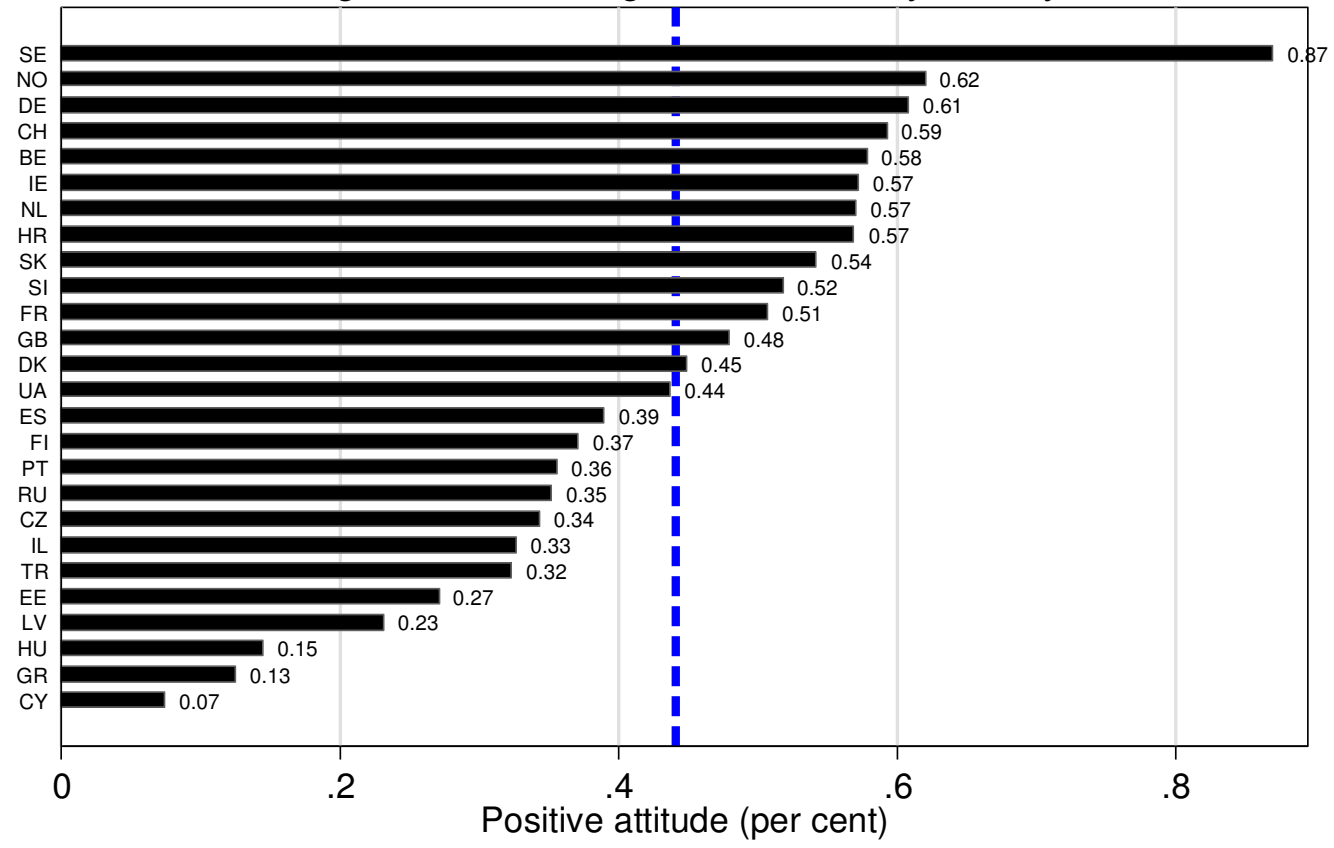

Notes: Weighted data using design weights.

Figure 3: Attitude towards immigration and public expenditure

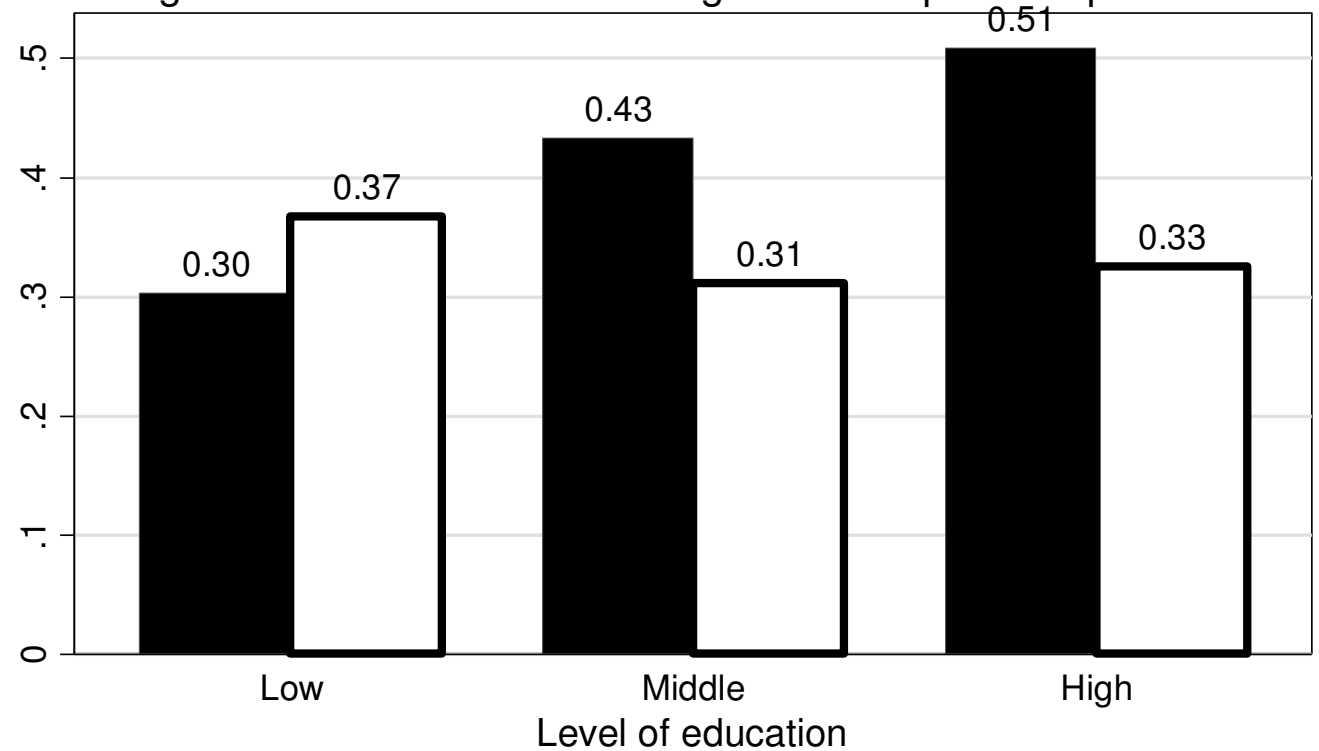

Pro-immigration $\square$ Pro-expenditure

Notes: Weighted data using both design and population weights. 
Figure 4: Proimm and Prospending by country type

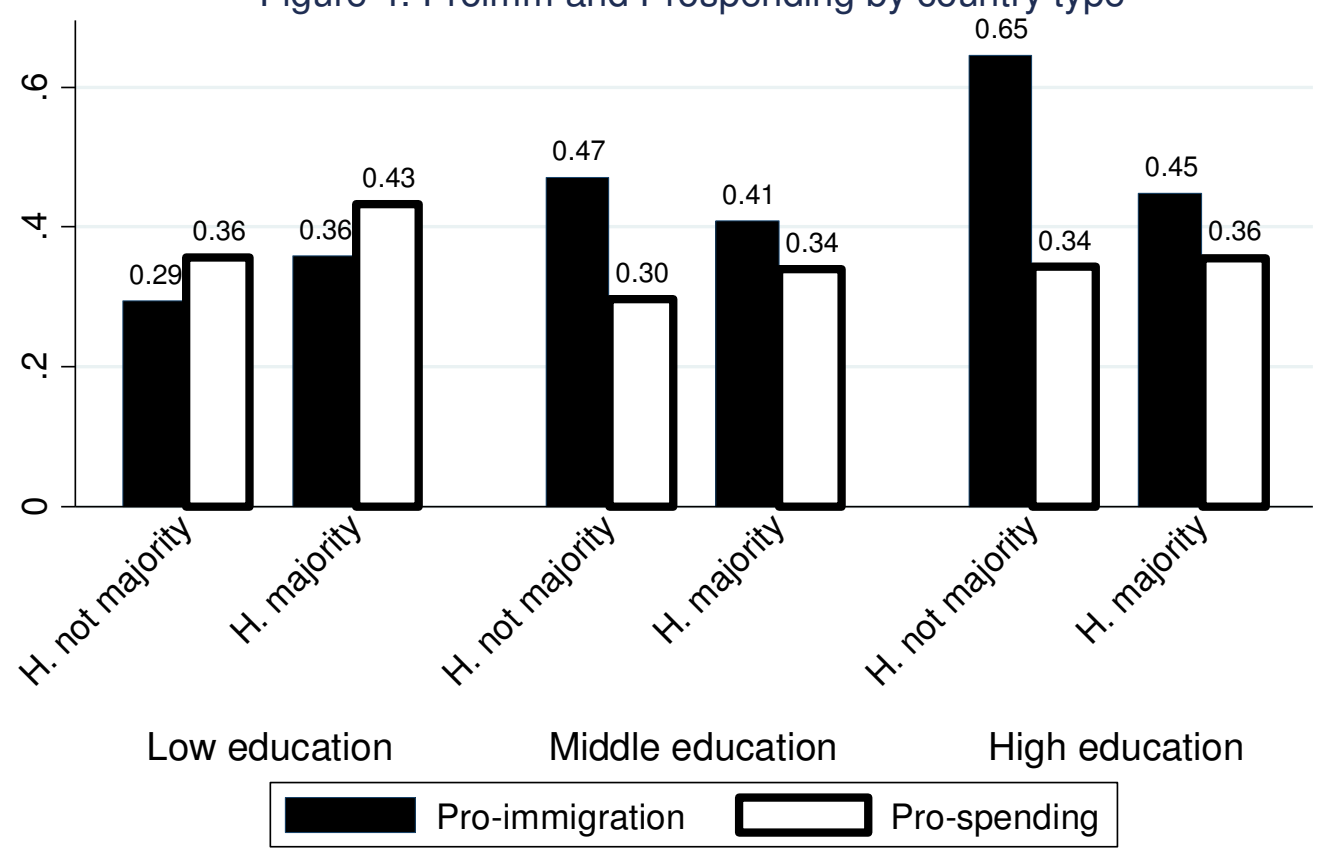

Notes: Weighted data using both design and population weights. 

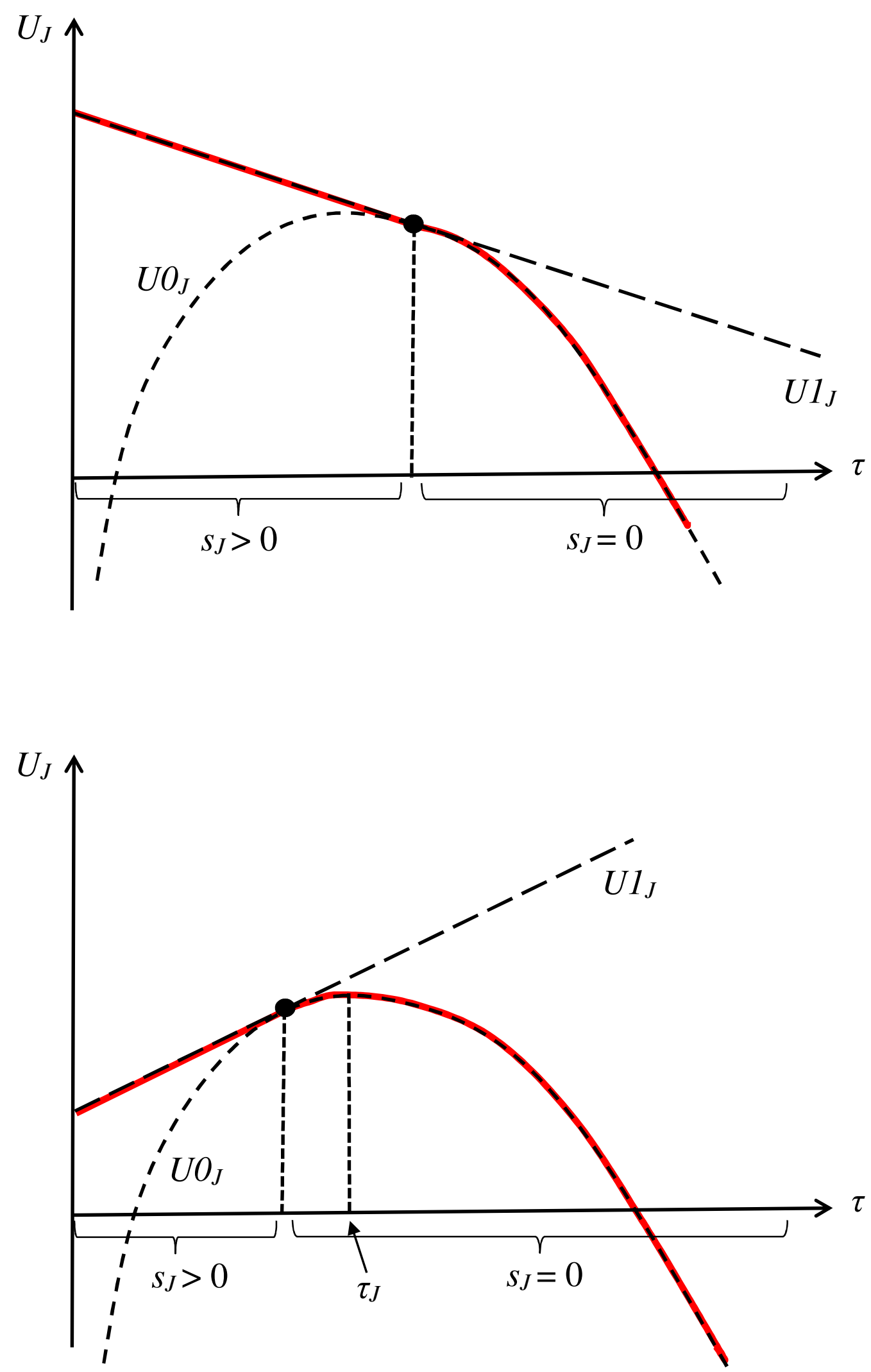

Figure A1: Illustration of Lemma 1 